\title{
ERROR ANALYSIS FOR MAPPED LEGENDRE SPECTRAL AND PSEUDOSPECTRAL METHODS*
}

\author{
JIE SHEN ${ }^{\dagger}$ AND LI-LIAN WANG W $^{\ddagger}$
}

\begin{abstract}
A general framework is introduced to analyze the approximation properties of mapped Legendre polynomials and of interpolations based on mapped Legendre-Gauss-Lobatto points. Optimal error estimates featuring explicit expressions on the mapping parameters for several popular mappings are derived. These results not only play an important role in numerical analysis of mapped Legendre spectral and pseudospectral methods for differential equations but also provide quantitative criteria for the choice of parameters in these mappings.
\end{abstract}

Key words. spectral approximation, mapped Legendre polynomials, interpolation, orthogonal system

AMS subject classifications. 65N35, 65N15, 65N50

DOI. $10.1137 /$ S0036142903422065

1. Introduction. In a spectral method, global polynomials are used as trial functions to approximate solutions of partial differential equations (PDEs); if the underlying solutions are smooth throughout the domain, the spectral method will provide very accurate approximations with significantly fewer degrees of freedom when compared with finite difference or finite element methods (cf. [13, 8, 7]). However, if the solutions of PDEs exhibit localized rapid variations such as spikes, sharp interfaces, or internal layers, standard spectral methods usually fail to produce accurate approximations with a reasonable number of degrees of freedom, for the grid is fixed in a standard spectral method and does not take into account the localized solution behaviors. Thus, for problems with localized rapid variations, it is advisable to use a grid adapted to the localized solution behaviors rather than a standard fixed grid.

However, unlike in a finite difference or finite element method, spectral methods cannot gracefully handle an arbitrarily locally refined grid, for the spectral accuracy will usually be lost due to the fact that the locally refined grid cannot, in general, be "smoothly" mapped to the standard spectral grid. Thus the adaptivity for spectral methods is best realized through a "smooth" map which transforms a function having sharp interfaces in the physical domain to a slow varying function on the computational domain. Hence two questions need to be addressed: (i) what is the influence of the mapping on the accuracy of the spectral methods? (ii) how do we adaptively determine a suitable mapping? In this paper, we aim to provide a complete answer to the first question, which is a first step toward a long-term goal of designing a robust adaptive spectral method for solving PDEs.

In general, a coordinate transformation takes the form

$$
x=g(y ; \lambda), \quad y \in[-1,1], \quad \lambda \in D_{\lambda},
$$

* Received by the editors January 27, 2003; accepted for publication (in revised form) July 5, 2003; published electronically March 3, 2004.

http://www.siam.org/journals/sinum/42-1/42206.html

${ }^{\dagger}$ Department of Mathematics, Purdue University, West Lafayette, IN 47907 (shen@math.purdue. edu). The work of this author is partially supported by NFS grants DMS-0074283 and DMS-0311915.

${ }^{\ddagger}$ Department of Mathematics, Purdue University, West Lafayette, IN 47907 (lwang@math.purdue. edu), and Department of Mathematics, Shanghai Normal University, Shanghai, P.R. China. The work of this author is partially supported by The Shanghai Natural Science Foundation N.00JC14057 and The Shanghai Natural Science Foundation for Youth N. 01QN85. 
such that

$$
g^{\prime}(y ; \lambda)>0, \quad g( \pm 1, \lambda)= \pm 1, \quad \lambda \in D_{\lambda}
$$

where $\lambda$ is a parameter vector and $D_{\lambda}$ is the feasible domain of $\lambda$, and ' denotes the derivative with respect to $y$ so $(1.1)$ maps the interval $[-1,1]$ univalently onto itself. Without loss of generality, we assume that the mapping (1.1) is explicitly invertible and denote

$$
y=g^{-1}(x ; \lambda):=h(x ; \lambda), \quad x, y \in[-1,1], \quad \lambda \in D_{\lambda} .
$$

Several interesting mappings have been proposed and implemented in practice. In particular, Kosloff and Tal-Ezer [20] introduced the one-parameter mapping

$$
x=g(y ; \lambda)=\frac{\arcsin (\lambda y)}{\arcsin \lambda}, \quad 0<\lambda<1 .
$$

This mapping stretches the Chebyshev-Gauss-Lobatto grid toward a uniform grid as $\lambda \rightarrow 1^{-}$. Bayliss et al. [3] used a mapped Chebyshev method to treat the boundary layer problem with the mapping

$$
x=g(y ; \lambda)=(4 / \pi) \arctan (\tan (\pi(y-1) / 4) / \lambda)+1, \quad \lambda>0 .
$$

The mapping clusters more and more points near $x=-1$ (resp., $x=1$ ) as $\lambda \rightarrow 0^{+}$ (resp., as $\lambda \rightarrow+\infty$ ). Bayliss and Turkel [4] introduced a two-parameter mapping

$$
x=g(y ; \lambda)=\lambda_{2}+\tan \left(a_{1}\left(y-a_{0}\right)\right) / \lambda_{1}, \quad \lambda_{1}>0, \quad-1 \leq \lambda_{2}<1,
$$

where $a_{0}$ and $a_{1}$ are chosen to satisfy (1.2). Here, as $\lambda_{1}$ increases, more and more points are clustered near $x=\lambda_{2}$. These mappings have been successfully used to treat some practical problems with localized rapid variations. We note that in [1], the authors used properties special to Chebyshev polynomials to derive some error estimates on projection and interpolation errors of the mapped Chebyshev methods with the mapping (1.3). However, as far as we know, there is neither a systematic framework for analyzing the mapped spectral methods for solving PDEs nor a precise rigorous analysis on how the mapping parameter(s) would affect the accuracy. For example, there have been controversies as to whether $\lambda$ (with $\lambda$ close to 1) in [20] would degrade the accuracy $[20,9,1,23]$.

The main purposes of this paper are: (i) to establish a general framework for analyzing the mapped Legendre spectral methods as a first step toward an efficient adaptive spectral method; (ii) to provide precise information on how the mapping parameters affect the accuracy of the mapped spectral method.

For a given mapping, there are essentially two approaches to implement (and analyze) a mapped spectral method. In the first approach, we use $x=g(y ; \lambda)$ to transform the original equation (with localized rapid variations in $x$ ) to a mapped equation (with smooth behaviors in $y$ ), and then apply a standard spectral method (in $y$ ) to the mapped equation (see, for instance, $[14,17]$ ). The main advantage of this approach is that standard spectral approximation results can be used for the analysis, but its main disadvantage is that the mapped equation is usually very complicated and its analysis is often cumbersome. In the second approach, we do not transform the equation, but we approximate its solution using a new family of orthogonal functions $\left\{p_{k}(h(x ; \lambda))\right\}$, which are obtained by applying the mapping $y=h(x ; \lambda)$ to classical 
orthogonal polynomials $\left\{p_{k}(y)\right\}$ (see, for instance, $[6,18,16]$ ) and which are suitable for capturing the localized rapid variations in the solution of the given problem. The analysis of this approach will require approximation results by using the new family of orthogonal functions. The advantage of this approach is that once these approximation results are established, it can be directly (i.e., without using a transform) applied to a large class of problems. We shall take the second approach and establish approximation results for the mapped Legendre polynomials. We emphasize that the two approaches will yield essentially the same approximate solutions (although the two implementations can be quite different). Hence the dependence of the error estimates on the mapping parameters established here for the second approach is essentially valid for the first approach.

The remainder of the paper is organized as follows. In the next section, we introduce the general framework for the mapped Legendre spectral and pseudospectral approximations. In section 3, we apply our general results to the specific mappings (1.3)-(1.5). In section 4, we consider the mapped Legendre approximations for a model problem and present some illustrative numerical results. Some concluding remarks are given in section 5 .

2. The general framework. In this section, we introduce a general framework for the error analysis of Legendre spectral methods using mapping (1.1) with (1.2). We assume that for a certain positive integer $r \geq 1$,

$$
h(x ; \lambda) \in C^{r}((-1,1)), \quad \lambda \in D_{\lambda} .
$$

2.1. Preliminaries. We first introduce some notation. Let $I=(-1,1)$, and let $\chi(x)>0$ be a given weight function on $I$. We define

$$
L_{\chi}^{2}(I)=\left\{v \mid v \text { is measurable on } I \text { and }\|v\|_{\chi}<\infty\right\},
$$

equipped with the following inner product and norm:

$$
(u, v)_{\chi}=\int_{I} u(x) v(x) \chi(x) d x, \quad\|v\|_{\chi}=(v, v)_{\chi}^{\frac{1}{2}} .
$$

The weighted Sobolev spaces $H_{\chi}^{m}(I)$ and $H_{0, \chi}^{m}(I)$ are defined as usual. The norm of $H_{\chi}^{m}(I)$ is defined as

$$
\|v\|_{m, \chi}=\left(\sum_{k=0}^{m}\left\|\partial_{x}^{k} v\right\|_{\chi}^{2}\right)^{\frac{1}{2}}
$$

In case $\chi(x) \equiv 1$, we shall drop the subscript $\chi$ in the notation for the sake of simplicity.

Let $\omega^{\alpha, \beta}(x)=(1-x)^{\alpha}(1+x)^{\beta}$ be the Jacobi weight function and $\mathbb{N}$ be the set of all nonnegative integers. For any $m \in \mathbb{N}$, we define the nonuniformly weighted Hilbert space

$$
A^{m}(I)=\left\{v \mid \partial_{x}^{k} v \in L_{\omega^{k, k}}^{2}(I), 0 \leq k \leq m\right\}
$$

equipped with the inner product, the seminorm, and the norm as follows:

$$
(u, v)_{m, A}=\sum_{k=0}^{m}\left(\partial_{x}^{k} u, \partial_{x}^{k} v\right)_{\omega^{k, k}}, \quad|v|_{m, A}=\left\|\partial_{x}^{m} v\right\|_{\omega^{m, m}}, \quad\|v\|_{m, A}=(v, v)_{m, A}^{\frac{1}{2}} .
$$


For any real $r>0$, we define the space $A^{r}(I)$ and its norm by space interpolation.

We shall use the expression $A \lesssim B$ to mean that there exist a generic positive constant $c$, independent of any function, $N$, and the parameters of the mappings, such that $A \leq c B$.

Let $L_{l}(y)$ be the Legendre polynomial of degree $l$, which is the eigenfunction of the Sturm-Liouville problem

$$
\partial_{y}\left(\left(1-y^{2}\right) \partial_{y} v(y)\right)+\mu v(y)=0, \quad y \in I,
$$

with the corresponding eigenvalues $\mu_{l}=l(l+1), l=0,1,2, \ldots$. We have $L_{l}( \pm 1)=$ $( \pm 1)^{l}$ and the following recurrence relations:

$$
\begin{array}{ll}
L_{l+1}(y)=\frac{2 l+1}{l+1} y L_{l}(y)-\frac{l}{l+1} L_{l-1}(y), & l \geq 1, \\
(2 l+1) L_{l}(y)=\partial_{y} L_{l+1}(y)-\partial_{y} L_{l-1}(y), & l \geq 1 .
\end{array}
$$

The set of Legendre polynomials forms an $L^{2}(I)$-orthogonal system, i.e.,

$$
\int_{I} L_{l}(y) L_{m}(y) d y=\gamma_{l} \delta_{l, m}, \quad \text { with } \quad \gamma_{l}=\frac{2}{2 l+1} .
$$

For any $v \in L^{2}(I)$, we write

$$
v(y)=\sum_{l=0}^{\infty} \hat{v}_{l} L_{l}(y), \quad \text { with } \quad \hat{v}_{l}=\frac{1}{\gamma_{l}}\left(v, L_{l}\right) .
$$

We have the following equivalence (see [17]):

$$
\left\|\partial_{y}^{r} v\right\|_{\omega^{r, r}} \sim\left(\sum_{l=r}^{\infty} \mu_{l}^{r} \hat{v}_{l}^{2} \gamma_{l}\right)^{\frac{1}{2}} \forall v \in A^{r}(I) .
$$

We now recall some results on the Legendre spectral approximations. Let $\mathcal{P}_{N}$ be the set of all polynomials of degree less than or equal to $N$ and $\mathcal{P}_{N}^{0}=\left\{\phi \in \mathcal{P}_{N}\right.$ : $\phi( \pm 1)=0\}$. We define $\widehat{P}_{N}: L^{2}(I) \rightarrow \mathcal{P}_{N}$ the $L^{2}(I)$-orthogonal projector by

$$
\left(\widehat{P}_{N} v-v, \phi\right)=0 \quad \forall \phi \in \mathcal{P}_{N} .
$$

The following result was proved in [11] (see also $[2,15]$ ).

LEMMA 2.1.

$$
\left\|\partial_{y}^{\mu}\left(\widehat{P}_{N} v-v\right)\right\|_{\omega^{\mu, \mu}} \lesssim N^{\mu-r}\left\|\partial_{y}^{r} v\right\|_{\omega^{r, r}}, \quad 0 \leq \mu \leq r, v \in A^{r}(I) .
$$

We define the $H^{1}(I)$-orthogonal projector $\widehat{P}_{N}^{1}: H^{1}(I) \rightarrow \mathcal{P}_{N}$ by

$$
\left(\widehat{P}_{N}^{1} v-v, \phi\right)_{1}=0 \quad \forall \phi \in \mathcal{P}_{N}
$$

and the $H_{0}^{1}(I)$-orthogonal projector $\widehat{P}_{N}^{1,0}: H_{0}^{1}(I) \rightarrow \mathcal{P}_{N}^{0}$ by

$$
\left(\partial_{y}\left(\widehat{P}_{N}^{1,0} v-v\right), \partial_{y} \phi\right)=0 \quad \forall \phi \in \mathcal{P}_{N}^{0} .
$$


As two special cases of Theorem 3.1 and Theorem 3.4 in Guo and Wang [19], we have the following lemma.

LeMma 2.2. If $v \in H^{1}(I)$ and $\partial_{y}^{r} v \in L_{\omega^{r-1, r-1}}^{2}(I)$, then

$$
\left\|\widehat{P}_{N}^{1} v-v\right\|_{\mu} \lesssim N^{\mu-r}\left\|\partial_{y}^{r} v\right\|_{\omega^{r-1, r-1}}, \quad 0 \leq \mu \leq 1 \leq r .
$$

If $v \in H_{0}^{1}(I)$ and $\partial_{y}^{r} v \in L_{\omega^{r-1, r-1}}^{2}(I)$, then

$$
\left\|\widehat{P}_{N}^{1,0} v-v\right\|_{\mu} \lesssim N^{\mu-r}\left\|\partial_{y}^{r} v\right\|_{\omega^{r-1, r-1}}, \quad 0 \leq \mu \leq 1 \leq r .
$$

Next, let $\zeta_{N, j}, 0 \leq j \leq N$, be the Legendre-Gauss-Lobatto (LGL) points, which are the zeros of $\left(1-y^{2}\right) \partial_{y} L_{N}(y)$. We assume that they are arranged in ascending order. There exists a unique set of Christoffel numbers $\left\{\omega_{N, j}\right\}$ such that

$$
\int_{I} \phi(y) d y=\sum_{j=0}^{N} \phi\left(\zeta_{N, j}\right) \omega_{N, j} \quad \forall \phi \in \mathcal{P}_{2 N-1} .
$$

In fact, we have

$$
\omega_{N, 0}=\omega_{N, N}=\frac{2}{N(N+1)}, \omega_{N, j}=\frac{2}{N(N+1)}\left(L_{N}\left(\zeta_{N, j}\right)\right)^{-2}, \quad 1 \leq j \leq N-1 .
$$

We define the discrete inner product and discrete norm as

$$
(u, v)_{N}=\sum_{j=0}^{N} u\left(\zeta_{N, j}\right) v\left(\zeta_{N, j}\right) \omega_{N, j}, \quad\|v\|_{N}=(v, v)_{N}^{\frac{1}{2}} .
$$

Note that we have (see, for instance, formula (21.8) of Bernardi and Maday [5])

$$
\|\phi\| \leq\|\phi\|_{N} \leq \sqrt{2+N^{-1}}\|\phi\| \quad \forall \phi \in \mathcal{P}_{N}
$$

On the other hand, we also have (see Theorem 4.9 of Guo and Wang [15])

$$
\|v\|_{N} \lesssim\|v\|+N^{-1}\left\|\partial_{y} v\right\|_{\omega^{1,1}} \quad \forall v \in H_{0}^{1}(I) .
$$

2.2. Mapped Legendre orthogonal approximations. For a given mapping $y=h(x ; \lambda)$, we define the mapped Legendre polynomials by

$$
\mathcal{L}_{l}^{(\lambda)}(x)=L_{l}(y)=L_{l}(h(x ; \lambda)), \quad l=0,1,2, \ldots
$$

Due to $h( \pm 1 ; \lambda)= \pm 1$, we have $\mathcal{L}_{l}^{(\lambda)}( \pm 1)=( \pm 1)^{l}$. We denote the weight function

$$
\omega_{\lambda}(x):=h^{\prime}(x ; \lambda)=\frac{1}{g^{\prime}(y ; \lambda)} .
$$

Thanks to (2.5), we have the recurrence relation

$$
(2 l+1) \omega_{\lambda}(x) \mathcal{L}_{l}^{(\lambda)}(x)=\partial_{x} \mathcal{L}_{l+1}^{(\lambda)}(x)-\partial_{x} \mathcal{L}_{l-1}^{(\lambda)}(x), \quad l \geq 1 .
$$


By virtue of $(2.6)$, the set $\left\{\mathcal{L}_{l}^{(\lambda)}\right\}_{l=0}^{\infty}$ forms a complete orthogonal system in $L_{\omega_{\lambda}}^{2}(I)$, and consequently, for any $v \in L_{\omega_{\lambda}}^{2}(I)$, we can write

$$
v(x)=\sum_{l=0}^{\infty} \hat{v}_{l}^{(\lambda)} \mathcal{L}_{l}^{(\lambda)}(x), \quad \text { with } \quad \hat{v}_{l}^{(\lambda)}=\frac{1}{\gamma_{l}}\left(v, \mathcal{L}_{l}^{(\lambda)}\right)_{\omega_{\lambda}} .
$$

Moreover, $\mathcal{L}_{l}^{(\lambda)}$ is the eigenfunction of the Sturm-Liouville problem

$$
w_{\lambda}^{-1}(x) \partial_{x}\left(\tilde{\omega}_{\lambda}(x) \partial_{x} \mathcal{L}_{l}^{(\lambda)}(x)\right)+\mu_{l} \mathcal{L}_{l}^{(\lambda)}(x)=0, \quad x \in I,
$$

with $\tilde{\omega}_{\lambda}(x)=\left(1-h^{2}(x ; \lambda)\right) \omega_{\lambda}^{-1}(x)$. This implies that $\left\{\partial_{x} \mathcal{L}_{l}^{(\lambda)}\right\}_{l=1}^{\infty}$ forms an orthogonal system in $L_{\tilde{\omega}_{\lambda}}^{2}(I)$, i.e.,

$$
\int_{I} \partial_{x} \mathcal{L}_{l}^{(\lambda)}(x) \partial_{x} \mathcal{L}_{m}^{(\lambda)}(x) \tilde{\omega}_{\lambda}(x) d x=\mu_{l} \gamma_{l} \delta_{l, m} .
$$

We now consider error estimates for approximations using the orthogonal system $\left\{\mathcal{L}_{l}^{(\lambda)}\right\}_{l=0}^{\infty}$. For $\lambda \in D_{\lambda}$, we set $\mathcal{V}_{N, \lambda}=\operatorname{span}\left\{\mathcal{L}_{0}^{(\lambda)}, \mathcal{L}_{1}^{(\lambda)}, \ldots, \mathcal{L}_{N}^{(\lambda)}\right\}$. Let $P_{N, \lambda}: L_{\omega_{\lambda}}^{2}(I) \rightarrow$ $\mathcal{V}_{N, \lambda}$ be the $L_{\omega_{\lambda}}^{2}(I)$-orthogonal projector defined by

$$
\left(P_{N, \lambda} v-v, \phi\right)_{\omega_{\lambda}}=0 \quad \forall \phi \in \mathcal{V}_{N, \lambda} .
$$

For clarity, the following notation will be used in what follows:

$$
V_{\lambda}(y)=v \circ g(y ; \lambda)=v(x), \quad \Phi_{\lambda}(y)=\phi \circ g(y ; \lambda)=\phi(x), \quad x, y \in I, \quad \lambda \in D_{\lambda} .
$$

For $\lambda \in D_{\lambda}$ and $r \in \mathbb{N}$, we define

$$
A_{\lambda}^{r}(I)=\left\{\left.v \in L_{\omega_{\lambda}}^{2}(I)|| v\right|_{A_{\lambda}^{r}}=\left\|\left(1-y^{2}\right)^{\frac{r}{2}} \partial_{y}^{r} V_{\lambda}\right\|<\infty, y=h(x ; \lambda)\right\}
$$

and

$$
B_{\lambda}^{r}(I)=\left\{\left.v \in L_{\omega_{\lambda}}^{2}(I)|| v\right|_{B_{\lambda}^{r}}=\left\|\left(1-y^{2}\right)^{\frac{r-1}{2}} \partial_{y}^{r} V_{\lambda}\right\|<\infty, y=h(x ; \lambda)\right\} .
$$

The following is a fundamental result for the mapped Legendre spectral approximations.

Theorem 2.1. For any $v \in A_{\lambda}^{r}(I), \lambda \in D_{\lambda}$, and $r \geq 1$,

$$
\left\|\partial_{x}\left(P_{N, \lambda} v-v\right)\right\|_{\tilde{\omega}_{\lambda}}+N\left\|P_{N, \lambda} v-v\right\|_{\omega_{\lambda}} \lesssim N^{1-r}|v|_{A_{\lambda}^{r}} .
$$

Moreover, for $r>1$,

$$
\left|P_{N, \lambda} v( \pm 1)-v( \pm 1)\right| \lesssim N^{1-r}|v|_{A_{\lambda}^{r}}
$$

Proof. For $v \in L_{\omega_{\lambda}}^{2}(I)$, we have $V_{\lambda} \in L^{2}(I)$, so we can write

$$
v(x)=\sum_{l=0}^{\infty} \hat{v}_{l}^{(\lambda)} \mathcal{L}_{l}^{(\lambda)}(x)=\sum_{j=0}^{\infty} \widehat{V}_{l}^{(\lambda)} L_{l}(y)=V_{\lambda}(y)
$$

with

$$
\hat{v}_{l}^{(\lambda)}=\frac{1}{\gamma_{l}}\left(v, \mathcal{L}_{l}^{(\lambda)}\right)_{\omega_{\lambda}}=\frac{1}{\gamma_{l}}\left(V_{\lambda}, L_{l}\right)=\widehat{V}_{l}^{(\lambda)} .
$$


Let $\widehat{P}_{N}$ and $P_{N, \lambda}$ be the projectors defined in (2.8) and (2.23). We derive from (2.30) and Lemma 2.1 with $\mu=0$ that

$$
\begin{gathered}
\left\|P_{N, \lambda} v-v\right\|_{\omega_{\lambda}}^{2}=\sum_{l=N+1}^{\infty}\left(\hat{v}_{l}^{(\lambda)}\right)^{2} \gamma_{l}=\sum_{l=N+1}^{\infty}\left(\widehat{V}_{l}^{(\lambda)}\right)^{2} \gamma_{l}=\left\|\widehat{P}_{N} V_{\lambda}-V_{\lambda}\right\|^{2} \\
\lesssim N^{-2 r}\left\|\left(1-y^{2}\right)^{\frac{r}{2}} \partial_{y}^{r} V_{\lambda}\right\|^{2}=N^{-2 r}|v|_{A_{\lambda}^{r}}^{2}
\end{gathered}
$$

On the other hand, since $\left\{\partial_{y} L_{l}\right\}$ is $L_{\omega^{1,1}}^{2}(I)$-orthogonal, we derive from (2.22), (2.30), and Lemma 2.1 with $\mu=1$ that

$$
\begin{aligned}
& \left\|\partial_{x}\left(P_{N, \lambda} v-v\right)\right\|_{\tilde{\omega}_{\lambda}}^{2}=\sum_{l=N+1}^{\infty} \mu_{l} \gamma_{l}\left(\hat{v}_{l}^{(\lambda)}\right)^{2}=\sum_{l=N+1}^{\infty} \mu_{l} \gamma_{l}\left(\widehat{V}_{l}^{(\lambda)}\right)^{2} \\
& =\left\|\partial_{y}\left(\widehat{P}_{N} V_{\lambda}-V_{\lambda}\right)\right\|_{\omega^{1,1}}^{2} \lesssim N^{2(1-r)}|v|_{A_{\lambda}^{r}}^{2} .
\end{aligned}
$$

Next, since $\left|\mathcal{L}_{l}^{(\lambda)}( \pm 1)\right|=1$, we derive from (2.7), (2.29), and (2.30) that

$$
\begin{gathered}
\left|P_{N, \lambda} v( \pm 1)-v( \pm 1)\right| \leq \sum_{l=N+1}^{\infty}\left|\widehat{V}_{l}^{(\lambda)}\right| \leq C_{N, r}\left(\sum_{l=N+1}^{\infty} \mu_{l}^{r}\left(\widehat{V}_{l}^{(\lambda)}\right)^{2} \gamma_{l}\right)^{\frac{1}{2}} \\
\lesssim C_{N, r}\left\|\partial_{y}^{r} V_{\lambda}\right\|_{\omega^{r, r}} \lesssim C_{N, r}|v|_{A_{\lambda}^{r},}
\end{gathered}
$$

where for $r>1$,

$$
C_{N, r}=\left(\sum_{l=N+1}^{\infty} \mu_{l}^{-r} \gamma_{l}^{-1}\right)^{\frac{1}{2}} \lesssim\left(\sum_{l=N+1}^{\infty} l^{1-2 r}\right)^{\frac{1}{2}} \lesssim\left(\int_{N}^{\infty} x^{1-2 r} d x\right)^{\frac{1}{2}} \lesssim N^{1-r} .
$$

The proof is complete.

When analyzing mapped Legendre spectral methods for numerical solutions of PDEs, we often need to consider the $H_{\omega_{\lambda}}^{1}(I)$-orthogonal projection $P_{N, \lambda}^{1}: H_{\omega_{\lambda}}^{1}(I) \rightarrow$ $\mathcal{V}_{N, \lambda}$ defined by

$$
\left(P_{N, \lambda}^{1} v-v, \phi\right)_{1, \omega_{\lambda}}=0 \quad \forall \phi \in \mathcal{V}_{N, \lambda} .
$$

Theorem 2.2. For any $v \in H_{\omega_{\lambda}}^{1}(I) \cap B_{\lambda}^{r}(I), \lambda \in D_{\lambda}$, and $r \geq 1$,

$$
\left\|P_{N, \lambda}^{1} v-v\right\|_{1, \omega_{\lambda}} \lesssim\left(d_{\lambda, 1}+1\right) N^{1-r}|v|_{B_{\lambda}^{r}},
$$

where $d_{\lambda, 1}=\max _{x \in \bar{I}}\left|\omega_{\lambda}(x)\right|$.

Proof. By (2.19) and (2.24),

$$
\begin{aligned}
\|\phi-v\|_{1, \omega_{\lambda}}^{2} & =\int_{I}\left(\partial_{y}\left(\Phi_{\lambda}(y)-V_{\lambda}(y)\right)\right)^{2}\left(\frac{d y}{d x}\right)^{2} d y+\int_{I}\left(\Phi_{\lambda}(y)-V_{\lambda}(y)\right)^{2} d y \\
& \leq\left(d_{\lambda, 1}+1\right)^{2}\left\|\Phi_{\lambda}-V_{\lambda}\right\|_{1}^{2} .
\end{aligned}
$$

Next, we take $\phi(x)=\Phi_{\lambda}(y)=\widehat{P}_{N}^{1} V_{\lambda}(y)$ in (2.33), where $\widehat{P}_{N}^{1}$ is defined in (2.10), and we obtain from the projection theorem and Lemma 2.2 that

$$
\begin{aligned}
\left\|P_{N, \lambda}^{1} v-v\right\|_{1, \omega_{\lambda}} & =\inf _{\phi \in \mathcal{V}_{N, \lambda}}\|\phi-v\|_{1, \omega_{\lambda}} \leq\left(d_{\lambda, 1}+1\right)\left\|\widehat{P}_{N}^{1} V_{\lambda}-V_{\lambda}\right\|_{1} \\
& \lesssim\left(d_{\lambda, 1}+1\right) N^{1-r}\left\|\partial_{y}^{r} V_{\lambda}\right\|_{\omega^{r-1, r-1}} \lesssim\left(d_{\lambda, 1}+1\right) N^{1-r}|v|_{B_{\lambda}^{r}}
\end{aligned}
$$


Next, we consider the bilinear form

$$
a_{\omega_{\lambda}}^{(\nu)}(u, v)=\nu_{1}\left(\partial_{x} u, \partial_{x}\left(v \omega_{\lambda}\right)\right)+\nu_{2}(u, v)_{\omega_{\lambda}}
$$

(where $\nu=\left(\nu_{1}, \nu_{2}\right)$ and $\left.\nu_{i}>0, i=1,2\right)$ associated to the mapped Legendre spectral approximation of the model elliptic equation

$$
-\mu_{1} v_{x x}+\mu_{2} v=f, \quad v( \pm 1)=0 .
$$

Due to the nonuniform weight function $\omega_{\lambda}(x)$, the bilinear form $a_{\omega_{\lambda}}^{(\nu)}(u, v)$ is not symmetric. We first study its continuity and coercivity.

Lemma 2.3. For any $u, v \in H_{\omega_{\lambda}}^{1}(I)$,

$$
a_{\omega_{\lambda}}^{(\nu)}(u, v) \leq \nu_{1}\left(d_{\lambda, 2}+1\right)|u|_{1, \omega_{\lambda}}\|v\|_{1, \omega_{\lambda}}+\nu_{2}\|u\|_{\omega_{\lambda}}\|v\|_{\omega_{\lambda}},
$$

where

$$
d_{\lambda, 2}=\max _{x \in \bar{I}}\left|\omega_{\lambda}^{-1}(x) \partial_{x} \omega_{\lambda}(x)\right|
$$

For any $v \in H_{0, \omega_{\lambda}}^{1}(I)$,

$$
a_{\omega_{\lambda}}^{(\nu)}(v, v) \geq \nu_{1}|v|_{1, \omega_{\lambda}}^{2}+\left(\nu_{2}-\frac{\nu_{1}}{2} d_{\lambda, 3}\right)\|v\|_{\omega_{\lambda}}^{2}
$$

where

$$
d_{\lambda, 3}=\max _{x \in \bar{I}}\left\{\omega_{\lambda}^{-1}(x) \partial_{x}^{2} \omega_{\lambda}(x)\right\}
$$

Proof. By the Cauchy inequality,

$$
\begin{aligned}
a_{\omega_{\lambda}}^{(\nu)}(u, v) & \leq \nu_{1}\left|\left(\partial_{x} u, \partial_{x} v\right)_{\omega_{\lambda}}+\left(\partial_{x} u, v \partial_{x} \omega_{\lambda}\right)\right|+\nu_{2}\left|(u, v)_{\omega_{\lambda}}\right| \\
& \leq \nu_{1}\left(|u|_{1, \omega_{\lambda}}|v|_{1, \omega_{\lambda}}+\max _{x \in \bar{I}}\left|\omega_{\lambda}^{-1}(x) \partial_{x} \omega_{\lambda}(x)\left\|\left.u\right|_{1, \omega_{\lambda}}\right\| v \|_{\omega_{\lambda}}\right)+\nu_{2}\|u\|_{\omega_{\lambda}}\|v\|_{\omega_{\lambda}}\right. \\
& \leq \nu_{1}\left(d_{\lambda, 2}+1\right)|u|_{1, \omega_{\lambda}}\|v\|_{1, \omega_{\lambda}}+\nu_{2}\|u\|_{\omega_{\lambda}}\|v\|_{\omega_{\lambda}} .
\end{aligned}
$$

On the other hand,

$$
\begin{aligned}
a_{\omega_{\lambda}}^{(\nu)}(v, v) & =\nu_{1}|v|_{1, \omega_{\lambda}}^{2}+\nu_{2}\|v\|_{\omega_{\lambda}}^{2}+\frac{\nu_{1}}{2} \int_{I} \partial_{x}\left(v^{2}(x)\right) \partial_{x} \omega_{\lambda}(x) d x \\
& =\nu_{1}|v|_{1, \omega_{\lambda}}^{2}+\nu_{2}\|v\|_{\omega_{\lambda}}^{2}-\frac{\nu_{1}}{2} \int_{I} v^{2}(x) \partial_{x}^{2} \omega_{\lambda}(x) d x \\
& \geq \nu_{1}|v|_{1, \omega_{\lambda}}^{2}+\left(\nu_{2}-\frac{\nu_{1}}{2} d_{\lambda, 3}\right)\|v\|_{\omega_{\lambda}}^{2} \cdot \quad \square
\end{aligned}
$$

This lemma indicates that if $\nu_{2}>\frac{\nu_{1}}{2} d_{\lambda, 3}$, then $\|v\|_{1, \omega_{\lambda}}:=\sqrt{a_{\omega_{\lambda}}^{(\nu)}(v, v)}$ is a norm for the space $H_{0, \omega_{\lambda}}^{1}(I)$.

Next, we set $\mathcal{V}_{N, \lambda}^{0}=H_{0, \omega_{\lambda}}^{1}(I) \cap \mathcal{V}_{N, \lambda}$ and define the orthogonal projector $P_{N, \lambda}^{1,0}$ : $H_{0, \omega_{\lambda}}^{1}(I) \rightarrow \mathcal{V}_{N, \lambda}^{0}$ by

$$
a_{\omega_{\lambda}}^{(\nu)}\left(P_{N, \lambda}^{1,0} v-v, \phi\right)=0 \quad \forall \phi \in \mathcal{V}_{N, \lambda}^{0}
$$


TheOREM 2.3. If $\nu_{2}>\frac{\nu_{1}}{2} d_{\lambda, 3}$, then for any $v \in H_{0, \omega_{\lambda}}^{1}(I) \cap B_{\lambda}^{r}(I), \lambda \in D_{\lambda}$, and $r \geq 1$,

$$
\left\|\left|P_{N, \lambda}^{1,0} v-v \|\right|_{1, \omega_{\lambda}} \lesssim\left(\nu_{1}^{\frac{1}{2}}\left(d_{\lambda, 1}+1\right)\left(d_{\lambda, 2}+1\right)^{\frac{1}{2}}+\nu_{2}^{\frac{1}{2}} N^{-1}\right) N^{1-r}|v|_{B_{\lambda}^{r}},\right.
$$

where $d_{\lambda, i}, i=1,2,3$, are the same as before.

Proof. By the projection theorem, (2.33), and (2.35),

$$
\begin{aligned}
\| \mid P_{N, \lambda}^{1,0} v & -v\left\|\left.\right|_{1, \omega_{\lambda}} ^{2}=\inf _{\phi \in \mathcal{V}_{N, \lambda}^{0}}\right\| \phi-v \|_{1, \omega_{\lambda}}^{2} \\
& \leq \nu_{1}\left(d_{\lambda, 2}+1\right)|\phi-v|_{1, \omega_{\lambda}}\|\phi-v\|_{1, \omega_{\lambda}}+\nu_{2}\|\phi-v\|_{\omega_{\lambda}}^{2} \\
& \leq \nu_{1}\left(d_{\lambda, 1}+1\right)^{2}\left(d_{\lambda, 2}+1\right)\left\|\Phi_{\lambda}-V_{\lambda}\right\|_{1}^{2}+\nu_{2}\left\|\Phi_{\lambda}-V_{\lambda}\right\|^{2} .
\end{aligned}
$$

Let $\widehat{P}_{N}^{1,0}$ be the $H_{0}^{1}(I)$-orthogonal projection as in Lemma 2.2. Hence, by taking $\phi(x)=\Phi_{\lambda}(y)=\widehat{P}_{N}^{1,0} V_{\lambda}(y)$ in (2.42), where $\widehat{P}_{N}^{1,0}$ is defined in (2.11), we can obtain the desired result thanks to Lemma 2.2 .

2.3. Mapped Legendre pseudospectral approximations. In this subsection, we consider the interpolation operator based on the mapped Legendre-GaussLobatto (MLGL) points.

Let $\left\{\zeta_{N, j}\right\}_{j=0}^{N}$ and $\left\{\omega_{N, j}\right\}_{j=0}^{N}$ be the LGL points and weights. The MLGL points and weights are defined by

$$
\xi_{N, j}^{(\lambda)}=g\left(\zeta_{N, j} ; \lambda\right), \quad \omega_{N, j}^{(\lambda)}=\omega_{N, j}, \quad 0 \leq j \leq N, \quad \lambda \in D_{\lambda}
$$

It is clear that $\xi_{N, 0}^{(\lambda)}=-1$ and $\xi_{N, N}^{(\lambda)}=1$, and thanks to (2.14),

$$
\int_{I} \phi(x) \omega_{\lambda}(x) d x=\int_{I} \phi(g(y ; \lambda)) d y=\sum_{j=0}^{N} \phi\left(\xi_{N, j}^{(\lambda)}\right) \omega_{N, j}^{(\lambda)} \quad \forall \phi \in \mathcal{V}_{2 N-1, \lambda} .
$$

Let the discrete inner product and discrete norm be defined as

$$
(u, v)_{\omega_{\lambda}, N}=\sum_{j=0}^{N} u\left(\xi_{N, j}^{(\lambda)}\right) v\left(\xi_{N, j}^{(\lambda)}\right) \omega_{N, j}^{(\lambda)}, \quad\|v\|_{\omega_{\lambda}, N}=(v, v)_{\omega_{\lambda}, N}^{\frac{1}{2}} .
$$

We have from (2.16) that

$$
\|\phi\|_{\omega_{\lambda}} \leq\|\phi\|_{\omega_{\lambda}, N} \leq\left(2+\frac{1}{N}\right)^{\frac{1}{2}}\|\phi\|_{\omega_{\lambda}} \quad \forall \phi \in \mathcal{V}_{N, \lambda}
$$

Let $\mathcal{I}_{N, \lambda} v$ be the interpolation of $v(x)$ in $\mathcal{V}_{N, \lambda}$ at points $\xi_{N, j}^{(\lambda)}$. We first establish a result on the stability of $\mathcal{I}_{N, \lambda}$.

Lemma 2.4. For any $v \in L_{\omega_{\lambda}}^{2}(I) \cap H_{\tilde{\omega}_{\lambda}}^{1}(I)$ and $\lambda \in D_{\lambda}$,

$$
\left\|\mathcal{I}_{N, \lambda} v\right\|_{\omega_{\lambda}} \lesssim\|v\|_{\omega_{\lambda}}+N^{-1}\left(|v(1)|+|v(-1)|+|v|_{1, \tilde{\omega}_{\lambda}}\right) .
$$

Proof. By (2.15), (2.43), and (2.45),

$$
\begin{aligned}
\left\|\mathcal{I}_{N, \lambda} v\right\|_{\omega_{\lambda}}^{2} & \leq\left\|\mathcal{I}_{N, \lambda} v\right\|_{\omega_{\lambda}, N}^{2}=\frac{2}{N(N+1)}\left(v^{2}(-1)+v^{2}(1)\right)+\sum_{j=1}^{N-1} v^{2}\left(\xi_{N, j}^{(\lambda)}\right) \omega_{N, j}^{(\lambda)} \\
& =\frac{2}{N(N+1)}\left(v^{2}(-1)+v^{2}(1)\right)+\sum_{j=1}^{N-1} V_{\lambda}^{2}\left(\zeta_{N, j}\right) \omega_{N, j} .
\end{aligned}
$$


Thanks to (2.17), we have from (2.24) that

$$
\begin{gathered}
\sum_{j=1}^{N-1} V_{\lambda}^{2}\left(\zeta_{N, j}\right) \omega_{N, j} \leq\left\|V_{\lambda}\right\|_{N}^{2} \lesssim\left\|V_{\lambda}\right\|^{2}+N^{-2}\left\|\partial_{y} V_{\lambda}\right\|_{\omega^{1,1}}^{2} \\
\lesssim\|v\|_{\omega_{\lambda}}^{2}+N^{-2}|v|_{1, \tilde{\omega}_{\lambda}}^{2} .
\end{gathered}
$$

This completes the proof.

Remark 2.1. For the treatment of nonlinear problems, we often need to estimate the terms such as $\mathcal{I}_{N, \lambda} \phi$ with $\phi \in \mathcal{V}_{M, \lambda}^{0}, M>N$. By the formula (5.9) in [5], we have the following inverse inequality:

$$
|\phi|_{1, \tilde{\omega}_{\lambda}}=\left|\Phi_{\lambda}\right|_{1, \omega^{1,1}} \leq \sqrt{2} N\left\|\Phi_{\lambda}\right\|=\sqrt{2} N\|\phi\|_{\omega_{\lambda}} \quad \forall \phi \in \mathcal{V}_{N, \lambda} .
$$

So we obtain from (2.46) that for any $\phi \in \mathcal{V}_{M, \lambda}^{0}$ and $\psi \in \mathcal{V}_{L, \lambda}^{0}$,

$$
\left\|\mathcal{I}_{N, \lambda} \phi\right\|_{\omega_{\lambda}} \lesssim\|\phi\|_{\omega_{\lambda}}+N^{-1}|\phi|_{1, \tilde{\omega}_{\lambda}} \lesssim\left(1+\frac{M}{N}\right)\|\phi\|_{\omega_{\lambda}} .
$$

On the other hand, by (2.45),

$$
\begin{aligned}
\left|(\phi, \psi)_{\omega_{\lambda}, N}\right| & =\left|\left(\mathcal{I}_{N, \lambda} \phi, \mathcal{I}_{N, \lambda} \psi\right)_{\omega_{\lambda}, N}\right| \leq\left\|\mathcal{I}_{N, \lambda} \phi\right\|_{\omega_{\lambda}, N}\left\|\mathcal{I}_{N, \lambda} \psi\right\|_{\omega_{\lambda}, N} \\
& \leq\left(2+\frac{1}{N}\right)\left\|\mathcal{I}_{N, \lambda} \phi\right\|_{\omega_{\lambda}}\left\|\mathcal{I}_{N, \lambda} \psi\right\|_{\omega_{\lambda}} \\
& \lesssim\left(1+\frac{M}{N}\right)\left(1+\frac{L}{N}\right)\|\phi\|_{\omega_{\lambda}}\|\psi\|_{\omega_{\lambda}} .
\end{aligned}
$$

The following is the main result on the MLGL interpolation.

Theorem 2.4. For any $v \in A_{\lambda}^{r}(I), \lambda \in D_{\lambda}$, and $r>1$,

$$
\left\|\partial_{x}\left(\mathcal{I}_{N, \lambda} v-v\right)\right\|_{\tilde{\omega}_{\lambda}}+N\left\|\mathcal{I}_{N, \lambda} v-v\right\|_{\omega_{\lambda}} \lesssim N^{1-r}|v|_{A_{\lambda}^{r}} .
$$

Proof. By (2.27), (2.28), and (2.46),

$$
\begin{aligned}
\| \mathcal{I}_{N, \lambda} v- & P_{N, \lambda} v\left\|_{\omega_{\lambda}}=\right\| \mathcal{I}_{N, \lambda}\left(P_{N, \lambda} v-v\right) \|_{\omega_{\lambda}} \\
\lesssim & \left\|P_{N, \lambda} v-v\right\|_{\omega_{\lambda}}+N^{-1}\left(\left|P_{N, \lambda} v(1)-v(1)\right|\right. \\
& \left.\quad+\left|P_{N, \lambda} v(-1)-v(-1)\right|+\left\|\partial_{x}\left(P_{N, \lambda} v-v\right)\right\|_{\tilde{\omega}_{\lambda}}\right) \\
\lesssim & N^{-r}|v|_{A_{\lambda}^{r}} .
\end{aligned}
$$

Due to (2.49), we obtain from (2.53) that

$$
\left|\mathcal{I}_{N, \lambda} v-P_{N, \lambda} v\right|_{1, \tilde{\omega}_{\lambda}} \lesssim N\left\|\mathcal{I}_{N, \lambda} v-P_{N, \lambda} v\right\|_{\omega_{\lambda}} \lesssim N^{1-r}|v|_{A_{\lambda}^{r}} .
$$

We then derive from (2.27) that

$$
\begin{gathered}
\left|\mathcal{I}_{N, \lambda} v-v\right|_{1, \tilde{\omega}_{\lambda}}+N\left\|\mathcal{I}_{N, \lambda} v-v\right\|_{\omega_{\lambda}} \leq\left|\mathcal{I}_{N, \lambda} v-P_{N, \lambda} v\right|_{1, \tilde{\omega}_{\lambda}}+N\left\|\mathcal{I}_{N, \lambda} v-P_{N, \lambda} v\right\|_{\omega_{\lambda}} \\
\quad+\left|P_{N, \lambda} v-v\right|_{1, \tilde{\omega}_{\lambda}}+N\left\|P_{N, \lambda} v-v\right\|_{\omega_{\lambda}} \lesssim N^{1-r}|v|_{A_{\lambda}^{r}} .
\end{gathered}
$$

Remark 2.2. As a direct consequence, we can estimate the difference between the continuous and discrete inner products. In fact, we deduce from (2.27), (2.44), (2.45), and (2.52) that for any $v \in A_{\lambda}^{r}(I)$ and $\phi \in \mathcal{V}_{N, \lambda}$ with $r>1$ and $\lambda \in D_{\lambda}$,

$$
\begin{aligned}
\left|(v, \phi)_{\omega_{\lambda}}-(v, \phi)_{\omega_{\lambda}, N}\right| & \\
& \leq\left|(v, \phi)_{\omega_{\lambda}}-\left(P_{N-1, \lambda} v, v\right)_{\omega_{\lambda}}\right|+\left|\left(P_{N-1, \lambda} v, \phi\right)_{\omega_{\lambda}, N}-\left(\mathcal{I}_{N, \lambda} v, \phi\right)_{\omega_{\lambda}, N}\right| \\
& \leq\left\|P_{N-1, \lambda} v-v\right\|_{\omega_{\lambda}}\|\phi\|_{\omega_{\lambda}}+\left\|P_{N-1, \lambda} v-\mathcal{I}_{N, \lambda} v\right\|_{\omega_{\lambda}, N}\|\phi\|_{\omega_{\lambda}, N} \\
& \lesssim N^{-r}|v|_{A_{\lambda}^{r}}\|\phi\|_{\omega_{\lambda}} .
\end{aligned}
$$


3. The upper bounds of $|\boldsymbol{v}|_{A_{\lambda}^{r}}$ and $|\boldsymbol{v}|_{B_{\lambda}^{r}}$. In this section, we provide upper bounds of $|v|_{A_{\lambda}^{r}}$ and $|v|_{B_{\lambda}^{r}}$ in terms of derivatives of $v(x)$ for the mappings (1.3)-(1.5). We also derive explicit bounds on the positive constants $d_{\lambda, i}(i=1,2,3)$ defined in the previous section. These bounds provide, in particular, explicit information on how the mapping parameters affect the accuracy of the mapped Legendre approximation.

3.1. The mapping (1.3). In this case, we have $D_{\lambda}=(0,1)$. The inverse of the mapping (1.3) is

$$
y=h(x ; \lambda)=\frac{\sin (a x)}{\lambda}, \quad a=\arcsin \lambda, \quad \lambda \in(0,1) .
$$

Moreover,

$$
\omega_{\lambda}(x)=\frac{d y}{d x}=\frac{a}{\lambda} \cos (a x)=\left(\frac{d x}{d y}\right)^{-1}=\frac{a}{\lambda} \sqrt{1-\lambda^{2} y^{2}} .
$$

Since $a \rightarrow \lambda$ as $\lambda \rightarrow 0, \frac{\lambda}{a}$ is uniformly bounded for $\lambda \in(0,1)$. For clarity, let $Q_{l}(y ; \lambda)$ be a polynomial of degree $l$ with respect to $y$. Then for any integer $k \geq 1$,

$$
\begin{aligned}
\frac{d^{k} x}{d y^{k}} & =\frac{\lambda}{a} \sum_{j=0}^{k-1}\left(\begin{array}{c}
k-1 \\
j
\end{array}\right)\left((1-\lambda y)^{-\frac{1}{2}}\right)^{(j)}\left((1+\lambda y)^{-\frac{1}{2}}\right)^{(k-j-1)} \\
& =\sum_{j=0}^{k-1} E_{j}^{k}(\lambda)(1-\lambda y)^{-\frac{1}{2}-j}(1+\lambda y)^{\frac{1}{2}+j-k} \\
& =\left(1-\lambda^{2} y^{2}\right)^{\frac{1}{2}-k} \sum_{j=0}^{k-1} E_{j}^{k}(\lambda)(1-\lambda y)^{k-1-j}(1+\lambda y)^{j} \\
& =\left(1-\lambda^{2} y^{2}\right)^{\frac{1}{2}-k} Q_{k-1}(y ; \lambda),
\end{aligned}
$$

where $E_{j}^{k}(\lambda)$ is a constant in terms of $j, k$, and $\lambda$. By direct calculations,

$$
\begin{aligned}
\partial_{y} V_{\lambda}(y) & =\partial_{x} v(x) \frac{d x}{d y}=\left(1-\lambda^{2} y^{2}\right)^{-\frac{1}{2}} Q_{0}(y ; \lambda) \partial_{x} v(x), \\
\partial_{y}^{2} V_{\lambda}(y)= & \partial_{x}^{2} v(x)\left(\frac{d x}{d y}\right)^{2}+\partial_{x} v(x) \frac{d^{2} x}{d y^{2}} \\
= & \left(1-\lambda^{2} y^{2}\right)^{-1} Q_{0}(y ; \lambda) \partial_{x}^{2} v(x)+\left(1-\lambda^{2} y^{2}\right)^{-\frac{3}{2}} Q_{1}(y ; \lambda) \partial_{x} v(x), \\
\partial_{y}^{3} V_{\lambda}(y)= & \partial_{x}^{3} v(x)\left(\frac{d x}{d y}\right)^{3}+3 \partial_{x}^{2} v(x) \frac{d x}{d y} \frac{d^{2} x}{d y^{2}}+\partial_{x} v(x) \frac{d^{3} x}{d y^{3}} \\
= & \left(1-\lambda^{2} y^{2}\right)^{-\frac{3}{2}} Q_{0}(y ; \lambda) \partial_{x}^{3} v(x)+\left(1-\lambda^{2} y^{2}\right)^{-2} Q_{1}(y ; \lambda) \partial_{x}^{2} v(x) \\
& \quad+\left(1-\lambda^{2} y^{2}\right)^{-\frac{5}{2}} Q_{2}(y ; \lambda) \partial_{x} v(x) .
\end{aligned}
$$

Thus an induction argument leads to

$$
\partial_{y}^{k} V_{\lambda}(y)=\sum_{j=1}^{k}\left(1-\lambda^{2} y^{2}\right)^{\frac{j}{2}-k} Q_{k-j}(y ; \lambda) \partial_{x}^{j} v(x), \quad k \geq 1,
$$

where $Q_{l}(y ; \lambda)(0 \leq l \leq k)$ are uniformly bounded for all $y \in \bar{I}$ and $\lambda \in(0,1)$. Then, by the definition of $|v|_{A_{\lambda}^{r}}$, we derive from (3.5) that for $r \geq 1$,

$$
|v|_{A_{\lambda}^{r}}^{2}=\left\|\partial_{y}^{r} V_{\lambda}\right\|_{\omega^{r, r}}^{2} \lesssim \sum_{j=1}^{r} \int_{I}\left(1-\lambda^{2} y^{2}\right)^{j-2 r}\left(1-y^{2}\right)^{r}\left(\partial_{x}^{j} v(x)\right)^{2} \omega_{\lambda}(x) d x,
$$


where $y=g(x ; \lambda) \in \bar{I}$. Since $1-y^{2} \leq 1-\lambda^{2} y^{2}$ and $A_{\lambda}^{0}(I)=L_{\omega_{\lambda}}^{2}(I)$, we conclude that

$$
|v|_{A_{\lambda}^{r}} \lesssim\left(1-\lambda^{2}\right)^{\frac{1}{2}-\frac{r}{2}}\|v\|_{r, \omega_{\lambda}}, \quad r \geq 0, \quad \lambda \in(0,1) .
$$

Similarly, we derive from by the definition of $B_{\lambda}^{r}(I)$ that

$$
|v|_{B_{\lambda}^{r}} \lesssim\left(1-\lambda^{2}\right)^{-\frac{r}{2}}\|v\|_{r, \omega_{\lambda}}, \quad r \geq 1, \quad \lambda \in(0,1) .
$$

Next, we derive from (3.1) and (3.2) that the values of $d_{\lambda, i}, 1 \leq i \leq 3$, in (2.32), (2.35), and (2.38) are

$$
d_{\lambda, 1}=\frac{a}{\lambda}, \quad d_{\lambda, 2}=\frac{a \lambda}{\sqrt{1-\lambda^{2}}}, \quad d_{\lambda, 3}=-a^{2} .
$$

In summary, we have proved the following results.

Corollary 3.1. For any $v \in H_{\omega_{\lambda}}^{r}(I), \lambda \in(0,1)$, and $r \geq 1$,

$$
\begin{gathered}
\left\|\partial_{x}\left(P_{N, \lambda} v-v\right)\right\|_{\tilde{\omega}_{\lambda}}+N\left\|P_{N, \lambda} v-v\right\|_{\omega_{\lambda}} \lesssim\left(1-\lambda^{2}\right)^{\frac{1}{2}-\frac{r}{2}} N^{1-r}\|v\|_{r, \omega_{\lambda}}, \\
\left\|P_{N, \lambda}^{1} v-v\right\|_{1, \omega_{\lambda}} \lesssim\left(1-\lambda^{2}\right)^{-\frac{r}{2}} N^{1-r}\|v\|_{r, \omega_{\lambda}},
\end{gathered}
$$

and for $r>1$,

$$
\left\|\partial_{x}\left(\mathcal{I}_{N, \lambda} v-v\right)\right\|_{\tilde{\omega}_{\lambda}}+N\left\|\mathcal{I}_{N, \lambda} v-v\right\|_{\omega_{\lambda}} \lesssim\left(1-\lambda^{2}\right)^{\frac{1}{2}-\frac{r}{2}} N^{1-r}\|v\|_{r, \omega_{\lambda}} .
$$

If $\nu_{1}, \nu_{2}>0$ and $v \in H_{0, \omega_{\lambda}}^{1}(I) \cap H_{\omega_{\lambda}}^{r}(I)$ with $r \geq 1$, then

$$
\left\|\left|P_{N, \lambda}^{1,0} v-v\right|\right\|_{1, \omega_{\lambda}} \lesssim\left(\nu_{1}^{\frac{1}{2}}\left(1-\lambda^{2}\right)^{-\frac{1}{4}}+\nu_{2}^{\frac{1}{2}} N^{-1}\right)\left(1-\lambda^{2}\right)^{-\frac{r}{2}} N^{1-r}\|v\|_{r, \omega_{\lambda}} .
$$

Remark 3.1. For $\lambda=0,(1.3)$ becomes the identity map. So we obtain the same results as in the standard Legendre case, i.e.,

$$
|v|_{A_{\lambda}^{r}} \lesssim\left\|\left(1-x^{2}\right)^{\frac{r}{2}} \partial_{x}^{r} v\right\|, \quad|v|_{B_{\lambda}^{r}} \lesssim\left\|\left(1-x^{2}\right)^{\frac{r-1}{2}} \partial_{x}^{r} v\right\|, \quad \lambda \rightarrow 0 .
$$

Remark 3.2. For $\lambda=1$, (1.3) becomes $y=\sin (\pi x / 2)$. This mapping has singularities at $x= \pm 1$, and therefore, (3.7)-(3.8) are no longer valid. However, we find from (3.1), (3.2), and (3.6) that

$$
\begin{aligned}
|v|_{A_{1}^{r}} & \lesssim\left(\sum_{j=1}^{r} \int_{I}\left(1-y^{2}\right)^{j-r}\left(\partial_{x}^{j} v(x)\right)^{2} \omega_{\lambda}(x) d x\right)^{\frac{1}{2}} \\
& \lesssim\left(\sum_{j=1}^{r} \int_{I}\left(1-x^{2}\right)^{j-r+\frac{1}{2}}\left(\partial_{x}^{j} v(x)\right)^{2} d x\right)^{\frac{1}{2}} .
\end{aligned}
$$

This implies that $|v|_{A_{1}^{r}}$ is bounded if $v \in H^{r}(I)$ and for some $\sigma<1$,

$$
\left(1-x^{2}\right)^{j-r+\frac{1}{2}}\left(\partial_{x}^{j} v(x)\right)^{2} \lesssim \frac{1}{\left(1-x^{2}\right)^{\sigma}} \quad \text { as } \quad|x| \rightarrow 1, \quad 1 \leq j \leq r .
$$

In particular, one can verify that (3.14) is satisfied if

$$
\partial_{x}^{j} v( \pm 1)=0, \quad 1 \leq j \leq r-2, v \in H^{r}(I) .
$$


Indeed, by the Hardy's inequality (see [12]), we have that for $\alpha<1$,

$$
\int_{I} u^{2}(x)\left(1-x^{2}\right)^{\alpha-1} d x \lesssim \int_{I} u^{2}(x)\left(1-x^{2}\right)^{\alpha-2} d x \lesssim \int_{I}\left(\partial_{x} u(x)\right)^{2}\left(1-x^{2}\right)^{\alpha} d x,
$$

provided that $u( \pm 1)=0$ and the right-hand side of the inequality is finite. Using this equality and the condition (3.15), we have for $1 \leq j \leq r-2$,

$$
\begin{aligned}
\int_{I}\left(\partial_{x}^{j} v(x)\right)^{2} & \left(1-x^{2}\right)^{j-r+\frac{1}{2}} d x \lesssim \int_{I}\left(\partial_{x}^{j+1} v(x)\right)^{2}\left(1-x^{2}\right)^{j-r+\frac{3}{2}} d x \\
& \lesssim \cdots \lesssim \int_{I}\left(\partial_{x}^{r-1} v(x)\right)^{2}\left(1-x^{2}\right)^{-\frac{1}{2}} d x .
\end{aligned}
$$

Then, by the inequality (13.5) in [5], we have that for $\alpha>1$,

$$
\int_{I} u^{2}(x)\left(1-x^{2}\right)^{\alpha-2} d x \lesssim \int_{I}\left(\left(\partial_{x} u(x)\right)^{2}+u^{2}(x)\right)\left(1-x^{2}\right)^{\alpha} d x,
$$

which implies that

$$
\int_{I}\left(\partial_{x}^{r-1} v(x)\right)^{2}\left(1-x^{2}\right)^{-\frac{1}{2}} d x \lesssim \int_{I}\left(\left(\partial_{x}^{r} v(x)\right)^{2}+\left(\partial_{x}^{r-1} v(x)\right)^{2}\right)\left(1-x^{2}\right)^{\frac{3}{2}} d x .
$$

A combination of the above estimates show that under the condition (3.15), we have

$$
|v|_{A_{1}^{r}} \lesssim\left(\left\|\left(1-x^{2}\right)^{\frac{3}{4}} \partial_{x}^{r-1} v\right\|+\left\|\left(1-x^{2}\right)^{\frac{1}{4}} \partial_{x}^{r} v\right\|\right) .
$$

Similar results can also be derived for $|v|_{B_{1}^{r}}$. The estimates indicate, in particular, that, for the mapping (1.3) with $\lambda=1$, the convergence rate of the mapped Legendre method is of order $r$ if (3.15) is satisfied. In particular, only a second-order convergence rate can be expected if the function does not vanish at the end-points.

Remark 3.3. If the parameter $\lambda$ was chosen as (cf. [1] and [10])

$$
\lambda=\lambda(N, \epsilon)=\operatorname{sech}\left(\frac{|\ln \epsilon|}{N}\right)=\frac{2}{\epsilon^{1 / N}+\epsilon^{-1 / N}} \sim 1-\frac{1}{2}\left(\ln ^{2} \epsilon\right) N^{-2} \quad \text { for } N \gg 1
$$

where $\epsilon$ is the desired accuracy, then we find from (3.7) and (3.8) that for any $v \in$ $H_{\omega_{\lambda}}^{r}(I)$,

$$
|v|_{A_{\lambda}^{r}} \sim(|\ln \epsilon|)^{1-r} N^{r-1}, \quad|v|_{B_{\lambda}^{r}} \sim(|\ln \epsilon|)^{-r} N^{r},
$$

which, along with Corollary 3.1, implies that

$$
\left\|P_{N, \lambda} v-v\right\|_{\omega_{\lambda}} \sim|\ln \epsilon|^{1-r} N^{-1}, \quad\left\|P_{N, \lambda}^{1} v-v\right\|_{1, \omega_{\lambda}} \sim|\ln \epsilon|^{-r} N .
$$

Thus a lower order $(<r)$ of accuracy is expected by choosing (3.17), except when $\epsilon$ and $N$ are such that $\epsilon \lesssim \exp (-\gamma N), \gamma>0$.

3.2. The mapping (1.4). In this case, $D_{\lambda}=\{\lambda \mid \lambda>0\}$, and (1.4) is

$$
\begin{gathered}
y=h(x ; \lambda)=(4 / \pi) \arctan (\lambda \tan (\pi(x-1) / 4))+1, \quad \lambda>0, \\
\omega_{\lambda}(x)=\frac{d y}{d x}=\frac{\lambda}{1+\left(\lambda^{2}-1\right) \sin ^{2}(\pi(x-1) / 4)} .
\end{gathered}
$$


In particular, $h(x ; \lambda)=x$ and $\omega_{\lambda}(x)=1$ for $\lambda=1$.

Let us denote

$$
C_{\lambda}=\left\{\begin{array}{ll}
\lambda^{-1}, & 0<\lambda \leq 1, \\
\lambda, & \lambda>1,
\end{array} \quad C_{1}=\left\{\begin{array}{ll}
1, & 0<\lambda \leq 1, \\
\lambda^{-2}, & \lambda>1,
\end{array} \quad C_{2}= \begin{cases}\lambda^{2}, & 0<\lambda \leq 1, \\
1, & \lambda>1 .\end{cases}\right.\right.
$$

We have $C_{1}, C_{2} \leq 1$ for $\lambda>0$, and by (3.19),

$$
\begin{aligned}
\frac{d x}{d y} & =\left(\cos ^{2}(\pi(x-1) / 4)+\lambda^{2} \sin ^{2}(\pi(x-1) / 4)\right) / \lambda \\
& =C_{\lambda}\left(C_{1} \cos ^{2}(\pi(x-1) / 4)+C_{2} \sin ^{2}(\pi(x-1) / 4)\right) .
\end{aligned}
$$

We set

$$
\mathcal{T}_{l}=\operatorname{span}\{\cos (k \pi(x-1) / 4), \quad \sin (k \pi(x-1) / 4), 1 \leq k \leq l\}, \quad l \in \mathbb{N} .
$$

For $j \geq 1$, we denote by $T_{k, j}(x)$ some functions in $\mathcal{T}_{l}$ with coefficients in terms of $C_{1}$ and $C_{2}$. Then, by (3.18) and (3.20),

$$
\begin{aligned}
& \partial_{y} V_{\lambda}(y)=\partial_{x} v(x) \frac{d x}{d y}=C_{\lambda} T_{2,1}(x) \partial_{x} v(x), \\
& \partial_{y}^{2} V_{\lambda}(y)=\frac{d\left(\partial_{y} V_{\lambda}(y)\right)}{d x} \frac{d x}{d y}=C_{\lambda}^{2}\left(T_{4,2}(x) \partial_{x}^{2} v(x)+T_{4,1}(x) \partial_{x} v(x)\right) .
\end{aligned}
$$

Hence, by an induction argument, we find that for $k \geq 1$,

$$
\partial_{y}^{k} V_{\lambda}(y)=C_{\lambda}^{k} \sum_{j=1}^{k} T_{k, j}(x) \partial_{x}^{j} v(x)
$$

where $T_{k, j}(x)(1 \leq j \leq k)$ are uniformly bounded for all $x \in \bar{I}$ and $\lambda>0$. Let

$$
\omega_{\lambda}^{(r)}(x):=\omega_{\lambda}(x)\left(1-y^{2}\right)^{r}=\omega_{\lambda}(x)\left(1-h^{2}(x ; \lambda)\right)^{r}\left(\lesssim \omega_{\lambda}(x), \quad x \in I, \lambda>0\right) .
$$

By (3.18) and (3.20),

$$
\lim _{x \rightarrow 1} \frac{1-h(x ; \lambda)}{1-x}=\lambda, \quad \lim _{x \rightarrow-1} \frac{1+h(x ; \lambda)}{1+x}=\lambda^{-1} .
$$

By virtue of (3.21) and the definitions of $|v|_{A_{\lambda}^{r}}$ and $|v|_{B_{\lambda}^{r}}$, we derive that

$$
|v|_{A_{\lambda}^{r}} \lesssim C_{\lambda}^{r}\|v\|_{r, \omega_{\lambda}^{(r)}}, \quad|v|_{B_{\lambda}^{r}} \leq C_{\lambda}^{r}\|v\|_{r, \omega_{\lambda}^{(r-1)}}, \quad \lambda>0 .
$$

Next, we deduce from (3.18)-(3.20) that the values of the constants $d_{\lambda, i}, 1 \leq i \leq$ 3 , are

$$
\begin{aligned}
& d_{\lambda, 1}=C_{\lambda}, \quad d_{\lambda, 2}=\frac{\pi / 4\left|\lambda^{2}-1 \| \sin (\pi(x-1) / 2)\right|}{\cos ^{2}(\pi(x-1) / 4)+\lambda^{2} \sin ^{2}(\pi(x-1) / 4)} \leq \frac{\pi\left|\lambda^{2}-1\right|}{4 \lambda}, \\
& d_{\lambda, 3}= \begin{cases}\max \left\{\frac{\pi^{2}}{8}\left(1-\lambda^{2}\right), S_{\lambda}\left(z_{0}\right)\right\} & \text { if } \quad 0<\lambda \leq e_{0}, \\
\frac{\pi^{2}}{8}\left(1-\lambda^{2}\right) & \text { if } \quad e_{0}<\lambda \leq 1, \\
\frac{\pi^{2}\left(\lambda^{2}-1\right)}{8 \lambda^{2}} & \text { if } 1<\lambda \leq e_{1}, \\
\max \left\{\frac{\pi^{2}\left(\lambda^{2}-1\right)}{8 \lambda^{2}}, S_{\lambda}\left(z_{0}\right)\right\} & \text { if } \lambda>e_{1},\end{cases}
\end{aligned}
$$


where $e_{0}=\sqrt{\frac{\sqrt{97}-5}{6}}, e_{1}=\sqrt{\frac{5}{3}}, z_{0}=\frac{5 \lambda^{2}-3}{3\left(1-\lambda^{4}\right)}$, and

$$
S_{\lambda}(z)=\frac{\pi^{2} b\left(-2 b z^{2}+(3 b-2) z+1\right)}{8(1-b z)^{2}}, \quad \text { with } \quad b=1-\lambda^{2} .
$$

The estimate on $d_{\lambda, 2}$ is derived using a simple inequality $a^{2}+b^{2} \geq 2 a b$. The estimate on $d_{\lambda, 3}$ is nontrivial and its derivation is given in Appendix A.

In summary, we obtained the following approximation results for mapping (1.4).

Corollary 3.2. For any $v \in H_{\omega_{\lambda}^{(r)}}^{r}(I), \lambda \in(0,1)$, and $r \geq 1$,

$$
\left\|\partial_{x}\left(P_{N, \lambda} v-v\right)\right\|_{\tilde{\omega}_{\lambda}}+N\left\|P_{N, \lambda} v-v\right\|_{\omega_{\lambda}} \lesssim C_{\lambda}^{r} N^{1-r}\|v\|_{r, \omega_{\lambda}^{(r)}},
$$

and for $r>1$,

$$
\left\|\partial_{x}\left(\mathcal{I}_{N, \lambda} v-v\right)\right\|_{\tilde{\omega}_{\lambda}}+N\left\|\mathcal{I}_{N, \lambda} v-v\right\|_{\omega_{\lambda}} \lesssim C_{\lambda}^{r} N^{1-r}\|v\|_{r, \omega_{\lambda}^{(r)}},
$$

while for any $v \in H_{\omega_{\lambda}^{(r-1)}}^{r}(I)$ and $r \geq 1$,

$$
\left\|P_{N, \lambda}^{1} v-v\right\|_{1, \omega_{\lambda}} \lesssim C_{\lambda}^{r+1} N^{1-r}\|v\|_{r, \omega_{\lambda}^{(r-1)}} .
$$

If, in addition, $\nu_{2}>\frac{\nu_{1}}{2} d_{\lambda, 3}$ and $v \in H_{0, \omega_{\lambda}}^{1}(I)$, then

$$
\left\|P_{N, \lambda}^{1,0} v-v\right\|_{1, \omega_{\lambda}} \lesssim\left(\nu_{1}^{\frac{1}{2}}\left(d_{\lambda, 1}+1\right)\left(d_{\lambda, 2}+1\right)^{\frac{1}{2}}+\nu_{2}^{\frac{1}{2}} N^{-1}\right) N^{1-r}\|v\|_{r, \omega_{\lambda}^{(r-1)}},
$$

where $d_{\lambda, i}, i=1,2,3$ are given in (3.23).

3.3. The mapping (1.5). In this case, $\lambda=\left(\lambda_{1}, \lambda_{2}\right)$ and $D_{\lambda}=\left\{\left(\lambda_{1}, \lambda_{2}\right) \mid \lambda_{1}>\right.$ $\left.0,-1 \leq \lambda_{2}<1\right\}$. The mapping (1.5) is explicitly invertible:

$$
y=h(x ; \lambda)=a_{0}+\arctan \left(\lambda_{1}\left(x-\lambda_{2}\right)\right) / a_{1} .
$$

The values of $a_{0}$ and $a_{1}$ are

$$
a_{0}=a_{0}(\lambda)=\frac{\kappa_{1}-\kappa_{2}}{\kappa_{1}+\kappa_{2}}, \quad a_{1}=a_{1}(\lambda)=\frac{\kappa_{1}+\kappa_{2}}{2},
$$

where

$$
\kappa_{1}=\arctan \left(\lambda_{1}\left(1+\lambda_{2}\right)\right), \quad \kappa_{2}=\arctan \left(\lambda_{1}\left(1-\lambda_{2}\right)\right) .
$$

With the above choice, we find that

$$
-1 \leq a_{0}<1, \quad 0<a_{1}<\frac{\pi}{2} .
$$

The weight functions are

$$
\begin{gathered}
\omega_{\lambda}(x)=\frac{d y}{d x}=\frac{\lambda_{1}}{a_{1}\left(1+\lambda_{1}^{2}\left(x-\lambda_{2}\right)^{2}\right)}=\left(\frac{d x}{d y}\right)^{-1}=\frac{\lambda_{1}}{a_{1}} \cos ^{2}\left(a_{1}\left(y-a_{0}\right)\right), \\
\tilde{\omega}_{\lambda}(x)=a_{1}^{-1} \lambda_{1}^{-1}\left(\kappa_{1}-q(x ; \lambda)\right)\left(\kappa_{2}+q(x ; \lambda)\right)\left(1+\lambda_{1}^{2}\left(x-\lambda_{2}\right)^{2}\right),
\end{gathered}
$$


where $q(x ; \lambda)=\arctan \left(\lambda_{1}\left(x-\lambda_{2}\right)\right)$.

For simplicity, we rewrite (3.32) as

$$
\frac{d x}{d y}=\frac{a_{1}\left(\lambda_{1}+1\right)^{2}}{\lambda_{1}}\left(\frac{1}{\left(\lambda_{1}+1\right)^{2}}+\frac{\lambda_{1}^{2}}{\left(\lambda_{1}+1\right)^{2}}\left(x-\lambda_{2}\right)^{2}\right):=C_{\lambda}\left(D_{1}+D_{2}\left(x-\lambda_{2}\right)^{2}\right) .
$$

Since $a_{1} \rightarrow \lambda_{1}$ as $\lambda_{1} \rightarrow 0$, we have that for all $\lambda \in D_{\lambda}$,

$$
C_{\lambda} \lesssim \lambda_{1}+1, \quad 0<C_{\lambda}^{-1}, D_{1}, D_{2} \leq 1 .
$$

Let us denote by $Q_{l}\left(x-\lambda_{2}\right)$ a polynomial of degree $l$ with respect to $x-\lambda_{2}$ with coefficients in terms of $D_{1}, D_{2}$, and $C_{\lambda}^{-1}$. Then, by (3.28) and (3.34),

$$
\begin{aligned}
& \partial_{y} V_{\lambda}(y)=\partial_{x} v(x) \frac{d x}{d y}=C_{\lambda} Q_{2}\left(x-\lambda_{2}\right) \partial_{x} v(x), \\
& \partial_{y}^{2} V_{\lambda}(y)=\frac{d\left(\partial_{y} V_{\lambda}(y)\right)}{d x} \frac{d x}{d y}=C_{\lambda}^{2}\left(Q_{4}\left(x-\lambda_{2}\right) \partial_{x}^{2} v(x)+Q_{3}\left(x-\lambda_{2}\right) \partial_{x} v(x)\right) .
\end{aligned}
$$

Hence, by an induction argument, we find that for $k \geq 1$,

$$
\partial_{y}^{k} V_{\lambda}(y)=C_{\lambda}^{k} \sum_{j=1}^{k} Q_{k+j}\left(x-\lambda_{2}\right) \partial_{x}^{j} v(x)
$$

where $Q_{k+j}\left(x-\lambda_{2}\right)(1 \leq j \leq k)$ are uniformly bounded for all $x \in \bar{I}$ and $\lambda \in D_{\lambda}$. Let us denote

$$
S_{\lambda}(x ; r):=\left(1-y^{2}\right)^{r}=\frac{1}{a_{1}^{2 r}}\left(\kappa_{2}-\arctan \left(\lambda_{1}\left(x-\lambda_{2}\right)\right)\right)^{r}\left(\kappa_{1}+\arctan \left(\lambda_{1}\left(x-\lambda_{2}\right)\right)\right)^{r} .
$$

We have $S_{\lambda}(x ; r) \leq 1$, and

$$
\begin{aligned}
& \lim _{x \rightarrow 1} \frac{\kappa_{2}-\arctan \left(\lambda_{1}\left(x-\lambda_{2}\right)\right)}{1-x}=\frac{\lambda_{1}}{1+\lambda_{1}^{2}\left(1-\lambda_{2}\right)^{2}}, \\
& \lim _{x \rightarrow-1} \frac{\kappa_{1}+\arctan \left(\lambda_{1}\left(x-\lambda_{2}\right)\right)}{1+x}=\frac{\lambda_{1}}{1+\lambda_{1}^{2}\left(1+\lambda_{2}\right)^{2}} .
\end{aligned}
$$

Consequently,

$$
\lim _{|x| \rightarrow 1} S_{\lambda}(x ; r)=G_{\lambda}^{r}\left(1-x^{2}\right)^{r} \quad \text { with } \quad G_{\lambda}=\frac{\lambda_{1}^{2}}{a_{1}^{2 r}\left(1+\lambda_{1}^{2}\left(1-\lambda_{2}\right)^{2}\right)\left(1+\lambda_{1}^{2}\left(1+\lambda_{2}\right)^{2}\right)} .
$$

Next, let

$$
\varpi_{\lambda}^{(r)}(x):=\omega_{\lambda}(x) S_{\lambda}(x ; r) \lesssim \omega_{\lambda}(x), \quad x \in I, \lambda \in D_{\lambda} .
$$

By the definition of $|v|_{A_{\lambda}^{r}}$ and (3.35),

$$
|v|_{A_{\lambda}^{r}} \lesssim\left(\lambda_{1}+1\right)^{r}\|v\|_{r, \varpi_{\lambda}^{(r)}}, \quad r \geq 0, \quad \lambda \in D_{\lambda} .
$$


Similarly, we deduce that

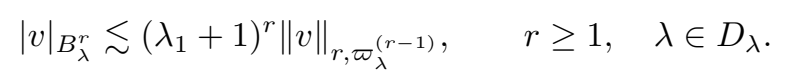

Remark 3.4. We find from (3.28)-(3.30) and (3.32) that

$$
a_{0} \rightarrow \lambda_{2}, \quad a_{1} \rightarrow 1, \quad h(x ; \lambda) \rightarrow x, \quad \omega_{\lambda}(x) \rightarrow 1, \quad \text { as } \quad \lambda_{1} \rightarrow 0 .
$$

So we have the same estimate as for the standard Legendre case:

$$
|v|_{A_{\lambda}^{r}} \lesssim\left\|(1-x)^{\frac{r}{2}} \partial_{x}^{r} v\right\|, \quad|v|_{B_{\lambda}^{r}} \lesssim\left\|(1-x)^{\frac{r-1}{2}} \partial_{x}^{r} v\right\| .
$$

Remark 3.5. We observe from the derivation of (3.36) that if the function $v$ possesses certain special properties as specified below, more precise estimates can be derived. For instance, if the rapid variational region of $v(x)$ is contained in $O_{\varepsilon}\left(\lambda_{2}\right):=$ $\left(\lambda_{2}-\varepsilon, \lambda_{2}+\varepsilon\right)$ for some $\varepsilon>0$, we can assume

$$
\sup _{x \in I_{\epsilon}}\left|\partial_{x}^{j} v(x)\right| \leq \delta(\varepsilon) C_{\lambda}^{-r}, \quad 0 \leq j \leq r
$$

where $I_{\varepsilon}=\bar{I} \backslash O_{\varepsilon}\left(\lambda_{2}\right)$, and $\delta(\varepsilon)$ is a small positive number corresponding to $\varepsilon$. Then

$$
|v|_{A_{\lambda}^{r}} \lesssim\left(\delta(\varepsilon)+a_{1}^{r} \lambda_{1}^{-r}\left(1+\lambda_{1}^{2} \varepsilon^{2}\right)^{r}\|v\|_{H_{\lambda}^{r}\left(O_{\varepsilon}^{r}\left(\lambda_{2}\right)\right)}\right) .
$$

In particular, if

$$
\operatorname{supp}\left\{\partial_{x}^{j} v(x)\right\} \subseteq \overline{O_{\varepsilon}\left(\lambda_{2}\right)} \subseteq[-1,1], \quad 0 \leq j \leq r,
$$

then we have

$$
|v|_{A_{\lambda}^{r}} \lesssim\left(a_{1}^{r} \lambda_{1}^{-r}\left(1+\lambda_{1}^{2} \varepsilon^{2}\right)^{r}\|v\|_{H_{\lambda}^{r(r)}\left(O_{\varepsilon}\left(\lambda_{2}\right)\right)}\right) .
$$

The above analysis is also valid for $|v|_{B_{\lambda}^{r}}$.

Next, we compute the values of $d_{\lambda, i}, i=1,2,3$. Using (3.28) and (3.32) yields

$$
d_{\lambda, 1}=a_{1} \lambda_{1}^{-1}, \quad d_{\lambda, 2}=\max _{x \in \bar{I}} \frac{2 \lambda_{1}^{2}\left|x-\lambda_{2}\right|}{1+\lambda_{1}^{2}\left(x-\lambda_{2}\right)^{2}} \leq \lambda_{1}, \quad d_{\lambda, 3} \leq \frac{3}{2} \lambda_{1}^{2} .
$$

The derivation of $d_{\lambda, 3}$ is a little complicated, so we defer it to Appendix B.

A combination of Theorems 2.1-2.4 and the above estimates leads to the following approximation results.

Corollary 3.3. For any $v \in H_{\varpi_{\lambda}^{(r)}}^{r}(I), \lambda \in D_{\lambda}$, and $r \geq 1$,

$$
\left\|\partial_{x}\left(P_{N, \lambda} v-v\right)\right\|_{\tilde{\omega}_{\lambda}}+N\left\|P_{N, \lambda} v-v\right\|_{\omega_{\lambda}} \lesssim\left(\lambda_{1}+1\right)^{r} N^{1-r}\|v\|_{r, \varpi_{\lambda}^{(r)}},
$$

and for $r>1$,

$$
\left\|\partial_{x}\left(\mathcal{I}_{N, \lambda} v-v\right)\right\|_{\tilde{\omega}_{\lambda}}+N\left\|\mathcal{I}_{N, \lambda} v-v\right\|_{\omega_{\lambda}} \lesssim\left(\lambda_{1}+1\right)^{r} N^{1-r}\|v\|_{r, \varpi_{\lambda}^{(r)}},
$$

while for any $v \in H_{\varpi_{\lambda}^{(r-1)}}^{r}(I)$ and $r \geq 1$,

$$
\left\|P_{N, \lambda}^{1} v-v\right\|_{1, \omega_{\lambda}} \lesssim\left(\lambda_{1}+1\right)^{r} N^{1-r}\|v\|_{r, \varpi_{\lambda}^{(r-1)}} .
$$

If, in addition, $\nu_{2}>\frac{\nu_{1}}{2} d_{\lambda, 3}$ and $v \in H_{0, \omega_{\lambda}}^{1}(I)$, then

$$
\left\|\left|P_{N, \lambda}^{1,0} v-v\right|\right\|_{1, \omega_{\lambda}} \lesssim\left(\nu_{1}^{\frac{1}{2}}\left(d_{\lambda, 1}+1\right)\left(d_{\lambda, 2}+1\right)^{\frac{1}{2}}+\nu_{2}^{\frac{1}{2}} N^{-1}\right) N^{1-r}\|v\|_{r, \varpi_{\lambda}^{(r-1)}},
$$

where $d_{\lambda, i}, i=1,2,3$, are given in $(3.41)$. 
3.4. Some other mappings. We consider here several useful mappings which do not quite fit into our framework.

Let us consider first the mapping

$$
C(s)=(1-b) \frac{\sinh (\theta s)}{\sinh (\theta)}+b \frac{\tanh (\theta(s+1 / 2))-\tanh (\theta / 2)}{2 \tanh (\theta / 2)}, s \in[-1,0],
$$

which was first introduced by Song and Haidvogel [26] as part of the so-called $s$ coordinates in their ocean circulation model. The two parameters $0 \leq \theta \leq 20$ and $0 \leq b \leq 1$ are used to fit the surface and bottom topography.

In order to apply our general framework, we set $s=\frac{y-1}{2}, x=2 C(s)+1, \lambda_{1}=\theta$, and $\lambda_{2}=b$ in (3.46) to get

$$
\begin{gathered}
x=g(y ; \lambda)=2\left(1-\lambda_{2}\right) \frac{\sinh \left(\lambda_{1}(y-1) / 2\right)}{\sinh \left(\lambda_{1}\right)}+\lambda_{2} \frac{\tanh \left(\lambda_{1} y / 2\right)-\tanh \left(\lambda_{1} / 2\right)}{\tanh \left(\lambda_{1} / 2\right)}+1, \\
y \in[-1,1], \quad \lambda_{1} \geq 0, \quad 0 \leq \lambda_{2} \leq 1 .
\end{gathered}
$$

Clearly, it maps the interval $[-1,1]$ univalently onto itself with $g( \pm 1 ; \lambda)= \pm 1$, and it is an identity mapping when $\lambda_{1}=0$. However, this mapping is not explicitly invertible. For simplicity, we consider only the special case $\lambda_{2}=0$ and denote $\lambda:=\lambda_{1}$. In this case, the weight function is

$$
\omega_{\lambda}(x)=\left(\frac{d x}{d y}\right)^{-1}=\frac{\sinh \lambda}{\lambda} \operatorname{sech}(\lambda(y-1) / 2)>0, \quad x, y \in I, \lambda>0 .
$$

One can verify readily that

$$
\frac{\tanh \lambda}{\lambda} \leq \omega_{\lambda}(x) \leq \frac{\sinh \lambda}{\lambda}, \quad x \in I, \lambda>0 .
$$

To estimate the corresponding upper bounds of $|v|_{A_{\lambda}^{r}}$ and $|v|_{B_{\lambda}^{r}}$ (cf. section 2), we can follow the same procedure as for the mapping (1.3). Since

$$
\frac{d^{k} x}{d y^{k}}=\frac{2}{\sinh \lambda}\left(\frac{\lambda}{2}\right)^{k}\left\{\begin{array}{cc}
\sinh (\lambda(y-1) / 2) & \text { if } k \text { is even, } \\
\cosh (\lambda(y-1) / 2) & \text { if } k \text { is odd }
\end{array}\right.
$$

we find

$$
\left|\frac{d^{k} x}{d y^{k}}\right| \lesssim \lambda^{k} \operatorname{coth} \lambda, \quad x \in \bar{I}, \lambda>0, k \geq 1
$$

As in the derivations of (3.4)-(3.6), we obtain that for any $v \in H_{\omega_{\lambda}}^{r}(I)$,

$$
|v|_{A_{\lambda}^{r}},\left.|v|_{B_{\lambda}^{r}} \lesssim \lambda^{r}(\operatorname{coth} \lambda)^{r}|| v\right|_{r, \omega_{\lambda}} .
$$

In view of the facts

$$
\operatorname{coth} \lambda \sim \lambda^{-1} \text { if } \lambda \ll 1, \quad \operatorname{coth} \lambda \sim 1 \text { if } \lambda \gg 1,
$$

we conclude that for small $\lambda$, this mapping is close to the identity mapping, while for large $\lambda$, an extra factor $\lambda^{r}$ appears in the error estimates. 
The mapping techniques have been successfully used in spectral methods to resolve boundary layers. For instance, the following mapping is used in [21] and [22]:

$$
x=g(y ; m)=-1+\sigma_{m} \int_{-1}^{y}\left(1-t^{2}\right)^{m} d t, \text { with } \sigma_{m}=2 / \int_{-1}^{1}\left(1-y^{2}\right)^{m} d y, \quad m \in \mathbb{N} .
$$

Clearly, we have $g( \pm 1 ; m)= \pm 1$, and

$$
\omega_{m}(x)=\sigma_{m}^{-1}\left(1-y^{2}\right)^{-m}, \quad x, y \in I .
$$

As $m$ increases, more and more Gauss-type collocation points are clustered near the end-points \pm 1 so it is suitable for resolving very thin boundary layers. However, the mapping is singular at the end-points, which implies in particular that $d_{\lambda, 1}=\infty$. The same is true for the iterated mappings introduced by Tang and Trummer [27],

$$
x_{0}=y, \quad x_{m}=\sin \left(\frac{\pi}{2} x_{m-1}\right), \quad m \geq 1,
$$

which are very effective mappings for problems with thin boundary layers. Hence we cannot directly apply our general framework to these mappings. Although it is possible to derive some special estimates as we did in Remark 3.2, the computations would be very tedious. However, we shall consider in a forthcoming paper the mapped Jacobi method in which we will be able to handle mappings with singularities at the end-points.

4. The mapped Legendre methods for a model equation. To illustrate how the results we developed in previous sections can be applied to analyze the mapped Legendre spectral and pseudospectral methods for PDEs, we consider the following model equation:

$$
\left\{\begin{array}{l}
-\varepsilon \partial_{x}^{2} u(x)+u(x)=f(x), \quad x \in I, \\
u( \pm 1)=0 .
\end{array}\right.
$$

Let $\nu_{1}=\varepsilon$ and $\nu_{2}=1$ and $\omega_{\lambda}(x), a_{\omega_{\lambda}}^{(\nu)}(\cdot, \cdot)$ be the same as in section 2. A weighted variational formulation for (4.1) is to find $u \in H_{0, \omega_{\lambda}}^{1}(I)$ such that

$$
a_{\omega_{\lambda}}^{(\nu)}(u, v)=(f, v)_{\omega_{\lambda}} \quad \forall v \in H_{0, \omega_{\lambda}}^{1}(I) .
$$

It is clear from Lemma 2.3 that, if $\varepsilon d_{\lambda, 3}<2$ and $f \in L_{\omega_{\lambda}}^{2}(I)$, (4.2) admits a unique solution.

4.1. Error estimates. The mapped Legendre spectral approximation for (4.2) is to find $u_{N} \in \mathcal{V}_{N, \lambda}^{0}$ such that

$$
a_{\omega_{\lambda}}^{(\nu)}\left(u_{N}, v_{N}\right)=\left(f, v_{N}\right)_{\omega_{\lambda}} \quad \forall v_{N} \in \mathcal{V}_{N, \lambda}^{0} .
$$

Let $P_{N, \lambda}^{1,0}$ be the projector as in Theorem 2.3. Then, by (2.40), (4.2), and (4.3),

$$
a_{\omega_{\lambda}}^{(\nu)}\left(u-u_{N}, v_{N}\right)=a_{\omega_{\lambda}}^{(\nu)}\left(P_{N, \lambda}^{1,0} u-u_{N}, v_{N}\right)=0 \quad \forall v_{N} \in \mathcal{V}_{N, \lambda}^{0} .
$$

As a consequence of Theorem 2.3, we have the following theorem. 
THEOREM 4.1. Let $u$ and $u_{N}$ be, respectively, the solutions of (4.2) and (4.3). If $\varepsilon d_{\lambda, 3}<2, u \in H_{0, \omega_{\lambda}}^{1}(I) \cap B_{\lambda}^{r}(I), \lambda \in D_{\lambda}$, and $r \geq 1$, then

$$
\left.||\left|u-u_{N}\right|\right|_{1, \omega_{\lambda}} \lesssim\left(\varepsilon^{\frac{1}{2}}\left(d_{\lambda, 1}+1\right)\left(d_{\lambda, 2}+1\right)^{\frac{1}{2}}+N^{-1}\right) N^{1-r}|u|_{B_{\lambda}^{r}},
$$

where $d_{\lambda, i}, i=1,2,3$, are the same as in Theorem 2.3. For the mappings (1.3)-(1.5), the upper bound of $|u|_{B_{\lambda}^{r}}$ and the values of $d_{\lambda, i}, i=1,2,3$, are given in section 3 .

Unlike in the standard Legendre-Galerkin method, where the linear system can be made sparse by choosing suitable basis functions [24], the linear system associated to (4.3) is in general full (unless a very special mapping is used), and furthermore, it is very costly to evaluate the entries of the linear system. Hence it is often convenient to use the mapped Legendre collocation method: find $u_{N} \in \mathcal{V}_{N, \lambda}^{0}$ such that

$$
-\varepsilon \partial_{x}^{2} u_{N}\left(\xi_{N, j}^{(\lambda)}\right)+u_{N}\left(\xi_{N, j}^{(\lambda)}\right)=f\left(\xi_{N, j}^{(\lambda)}\right), \quad 1 \leq j \leq N-1,
$$

where $\left\{\xi_{N, j}^{(\lambda)}\right\}_{j=0}^{N}$ are the mapped LGL points defined in (2.43). Taking the discrete inner product of (4.5) with any $v_{N} \in \mathcal{V}_{N, \lambda}^{0}$, thanks to (2.44), we find that (4.5) is equivalent to the following: find $u_{N} \in \mathcal{V}_{N, \lambda}^{0}$ such that

$$
\varepsilon\left(\partial_{x} u_{N}, \partial_{x}\left(\omega_{\lambda} v_{N}\right)\right)+\left(u_{N}, v_{N}\right)_{\omega_{\lambda}, N}=\left(f, v_{N}\right)_{\omega_{\lambda}, N} \quad \forall v_{N} \in \mathcal{V}_{N, \lambda}^{0} .
$$

We note that the linear system associated with the above formulation is full and ill conditioned. However, as demonstrated in [24, 25], it can be efficiently solved by using a preconditioned conjugate gradient-type iterative method with the standard Legendre-Galerkin method for (4.1) as a preconditioner. Note that with the collocation approach, there is no additional cost involved if the original PDE (4.1) has variable coefficients.

THEOREM 4.2. Let $u$ and $u_{N}$ be, respectively, the solutions of (4.2) and (4.6). If $\varepsilon d_{\lambda, 3}<2, u \in H_{0, \omega_{\lambda}}^{1}(I) \cap B_{\lambda}^{r}(I)$, and $f \in A_{\lambda}^{s}(I), \lambda \in D_{\lambda}$ with $r \geq 1$ and $s>1$, then

$$
||\left|u-u_{N}\right|||_{1, \omega_{\lambda}} \lesssim\left(\varepsilon^{\frac{1}{2}}\left(d_{\lambda, 1}+1\right)\left(d_{\lambda, 2}+1\right)^{\frac{1}{2}}+N^{-1}\right) N^{1-r}|u|_{B_{\lambda}^{r}}+N^{-s}|f|_{A_{\lambda}^{s}},
$$

where $d_{\lambda, i}, i=1,2,3$, are the same as in Theorem 2.3. For the mappings (1.3)-(1.5), the upper bounds of $|u|_{B_{\lambda}^{r}},|f|_{A_{\lambda}^{s}}$ and the values of $d_{\lambda, i}, i=1,2,3$, are given in section 3 .

Proof. Let $U_{N}=P_{N, \lambda}^{1,0} u$ and $\hat{e}_{N}=U_{N}-u_{N}$. Then by (2.40), (4.2), and (4.6),

$$
\begin{gathered}
\varepsilon\left(\partial_{x} \hat{e}_{N}, \partial_{x}\left(\omega_{\lambda} v_{N}\right)\right)+\left(\hat{e}_{N}, v_{N}\right)_{\omega_{\lambda}, N}=\left(U_{N}, v_{N}\right)_{\omega_{\lambda}, N}-\left(U_{N}, v_{N}\right)_{\omega_{\lambda}} \\
+\left(f, v_{N}\right)_{\omega_{\lambda}}-\left(f, v_{N}\right)_{\omega_{\lambda}, N} \quad \forall v_{N} \in \mathcal{V}_{N, \lambda}^{0} .
\end{gathered}
$$

Taking $v_{N}=\hat{e}_{N}$ in (4.8), we have from (2.45) that

$$
\begin{aligned}
& a_{\lambda}^{(\nu)}\left(\hat{e}_{N}, \hat{e}_{N}\right) \leq \varepsilon\left(\partial_{x} \hat{e}_{N}, \partial_{x}\left(\omega_{\lambda} \hat{e}_{N}\right)\right)+\left\|\hat{e}_{N}\right\|_{\omega_{\lambda}, N}^{2} \\
& \quad \leq\left|\left(U_{N}, \hat{e}_{N}\right)_{\omega_{\lambda}, N}-\left(U_{N}, \hat{e}_{N}\right)_{\omega_{\lambda}}\right|+\left|\left(f, \hat{e}_{N}\right)_{\omega_{\lambda}}-\left(f, \hat{e}_{N}\right)_{\omega_{\lambda}, N}\right| .
\end{aligned}
$$

By Remark 2.3,

$$
\left|\left(f, \hat{e}_{N}\right)_{\omega_{\lambda}}-\left(f, \hat{e}_{N}\right)_{\omega_{\lambda}, N}\right| \lesssim N^{-s}|f|_{A_{\lambda}^{s}}\left\|\hat{e}_{N}\right\|_{\omega_{\lambda}}
$$




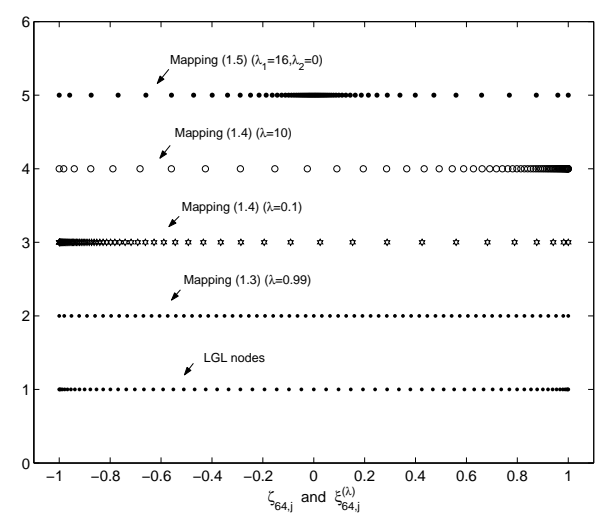

FIG. 1. $L G L$ vs. $M L G L$ points.

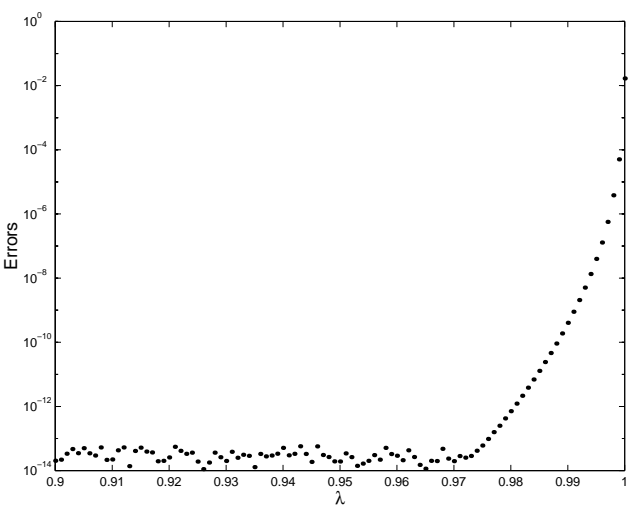

FIG. 2. Errors for mapping (1.3).

Moreover, by (2.44), (2.45), and Theorem 2.3,

$$
\begin{aligned}
& \left|\left(U_{N}, \hat{e}_{N}\right)_{\omega_{\lambda}, N}-\left(U_{N}, \hat{e}_{N}\right)_{\omega_{\lambda}}\right| \leq\left|\left(U_{N}-P_{N-1, \lambda}^{1,0} u, \hat{e}_{N}\right)_{\omega_{\lambda}, N}\right|+\left|\left(U_{N}-P_{N-1, \lambda}^{1,0} u, \hat{e}_{N}\right)_{\omega_{\lambda}}\right| \\
& \quad \lesssim\left\|U_{N}-P_{N-1, \lambda}^{1,0} u\right\|_{\omega_{\lambda}}\left\|e_{N}\right\|_{\omega_{\lambda}} \lesssim\left(\left.||\left|U_{N}-u\right|\right|_{1, \omega_{\lambda}}+\left.\left|\| P_{N-1, \lambda}^{1,0} u-u\right|\right|_{1, \omega_{\lambda}}\right)\left\|\hat{e}_{N}\right\|_{\omega_{\lambda}} \\
& \quad \lesssim\left(\varepsilon^{\frac{1}{2}}\left(d_{\lambda, 1}+1\right)\left(d_{\lambda, 2}+1\right)^{\frac{1}{2}}+N^{-1}\right) N^{1-r}|u|_{B_{\lambda}^{r}}\left\|\hat{e}_{N}\right\|_{\omega_{\lambda}} .
\end{aligned}
$$

The desired results follow from the above estimates.

4.2. Numerical results. We now present some numerical results with emphasis on how the accuracy depends on the choice of the parameters in the mappings (1.3)(1.5).

We first illustrate the effects of the parameters on the distributions of the MLGL points. In Figure 1, we plot the LGL points vs. the MLGL points $(N=64)$ with several typical parameters. It is clear that the mapping (1.3) stretches the grid evenly as $\lambda \rightarrow 1$; the mapping (1.4) clusters the points to $x=-1$ (resp., $x=1$ ) for $\lambda<1$ (resp., $\lambda>1$ ); and the mapping (1.5) clusters the points to $x=\lambda_{2}$ for $\lambda_{1}>1$.

Example 1. We consider (4.1) with the exact solution

$$
u(x)=\frac{e^{(1+x) / \sqrt{\varepsilon}}-e^{-(1+x) / \sqrt{\varepsilon}}}{e^{2 / \sqrt{\varepsilon}}-e^{-2 / \sqrt{\varepsilon}}}-\frac{1+x}{2} .
$$

This solution exhibits a boundary layer of width $O(\sqrt{\varepsilon})$ at $x=-1$.

We first take $\varepsilon=0.1$ so the solution is smoothly varying throughout the domain. We use (4.5) with the mapping (1.3) and $N=100$ to approximate (4.1). In Figure 2 , we plot the maximum absolute errors between $u$ and $u_{N}$ at the MLGL points with $\lambda \in[0.9,1]$. We see that the error increases very quickly as $\lambda \rightarrow 1$, which is in agreement with the theoretic analysis in Corollary 3.1 and Theorem 4.2. Hence it is not advisable to use mapping (1.3) with $\lambda$ close to 1.

Next we take $\varepsilon=10^{-8}$ so the solution has a thin boundary layer at $x=-1$, and we use (4.5) with the mapping (1.4) and $N=100$ to approximate (4.1). We plot in Figure 3 the errors with $\lambda \in[20,200]$. The results indicate that the errors grow as $\lambda$ increases, as predicted by Corollary 3.2 and Theorem 4.2 .

Example 2. We take $u(x)=\tanh (a x)$ with $a=150$. This solution has a large derivative at $x=0$; see Figure 4. We use (4.5) with mapping (1.5) and $N=100$. In 

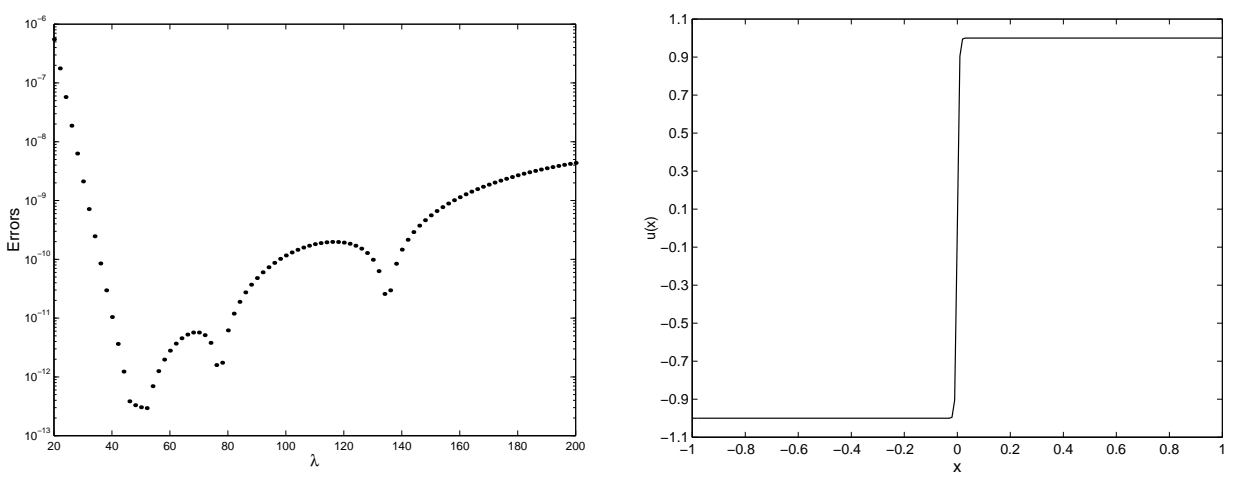

FiG. 3. Errors for mapping (1.4).

FIG. 4. Solution in Example 2.
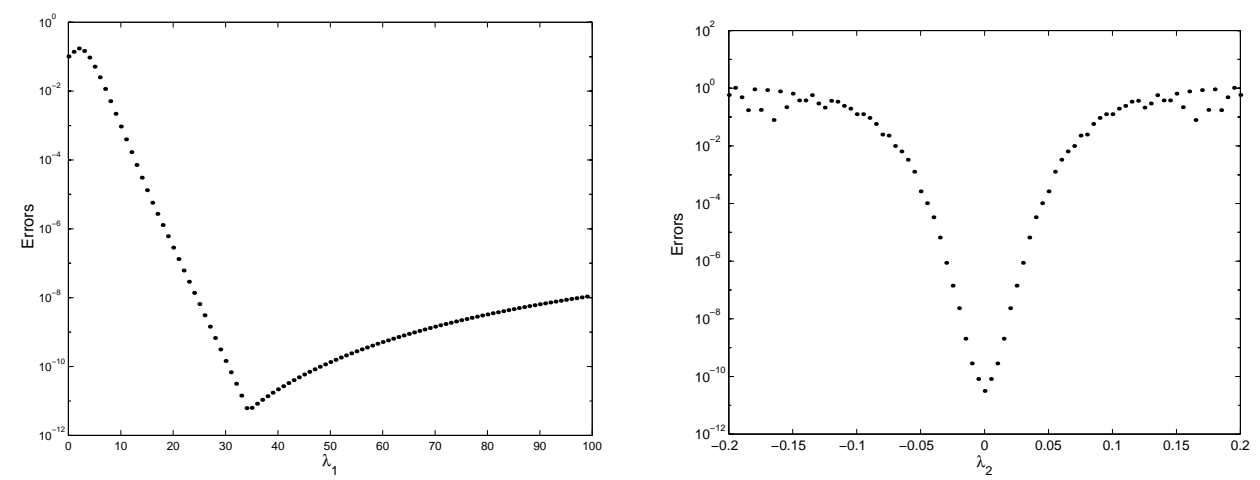

FIG. 5. Errors for mapping (1.5) with $\lambda_{2}=0$ and various $\lambda_{1}$ (left), and with $\lambda_{1}=51$ and various $\lambda_{2}$ (right).

Figure 5, we plot the maximum absolute errors at the MLGL points with $\lambda_{2}=0$ and various $\lambda_{1}$ (left panel) and with $\lambda_{1}=51$ and different $\lambda_{2}$ (right panel). Note that the accuracy is very sensitive to the choice of the parameter $\lambda_{2}$, which should be at the location of large variation, but less sensitive to the values of $\lambda_{1}$, which represents the intensity of the mapping at $x=\lambda_{2}$. Again, the numerical results are in agreement with Corollary 3.3 and Theorem 4.2.

5. Concluding remarks. We presented a general framework for analyzing the approximation properties of mapped Legendre polynomials and of interpolations based on MLGL points and derived optimal error estimates for general mappings. More precisely, we introduced a new family of orthogonal functions which are obtained by applying the mapping to Legendre polynomials, and we analyzed various projection and interpolation operators based on these mapped Legendre functions.

As an application of our general results, we considered the popular mappings (1.3)-(1.5) introduced in [20, 3, 4] and derived error estimates featuring explicit expressions on the mapping parameters. We used a model equation to show that these results not only play an important role in numerical analysis of mapped Legendre spectral and pseudospectral methods for differential equations but also provide quantitative criteria for the choice of parameters in these mappings.

This paper is a first step toward a long-term goal of designing a robust adaptive spectral method for solving PDEs. 
Appendix A. The derivation of $\boldsymbol{d}_{\boldsymbol{\lambda}, 3}$ in (3.23). For $\lambda=1$, we have $d_{\lambda, 3} \equiv 0$. We next consider $\lambda \neq 1$. For simplicity, let $z=\sin ^{2}(\pi(x-1) / 4)$ and $b=1-\lambda^{2}$. By (3.19) and a direct calculation, we find that

$$
S_{\lambda}(z):=\omega_{\lambda}^{-1}(x) \partial_{x}^{2} \omega_{\lambda}(x)=\frac{\pi^{2} b\left(-2 b z^{2}+(3 b-2) z+1\right)}{8(1-b z)^{2}}
$$

and

$$
S_{\lambda}^{\prime}(z)=\frac{\pi^{2} b(3 b(b-2) z+5 b-2)}{8(1-b z)^{3}} .
$$

Let us denote

$$
z_{0}=-\frac{5 b-2}{3 b(b-2)}=\frac{5 \lambda^{2}-3}{3\left(1-\lambda^{4}\right)}, \quad e_{0}=\sqrt{\frac{\sqrt{97}-5}{6}}, \quad e_{1}=\sqrt{\frac{5}{3}} .
$$

We find that

$$
\text { if } 0<\lambda \leq e_{0} \quad \text { or } \quad \lambda \geq e_{1} \text {, then }\left|z_{0}\right| \leq 1 \text {. }
$$

Hence

$$
\begin{aligned}
d_{\lambda, 3}= & \max _{z \in[0,1]} S_{\lambda}(z)=\max \left\{S_{\lambda}(0), S_{\lambda}\left(z_{0}\right), S_{\lambda}(1)\right\} \\
& =\left\{\begin{array}{lll}
\max \left\{\frac{\pi^{2}}{8}\left(1-\lambda^{2}\right), S_{\lambda}\left(z_{0}\right)\right\} & \text { if } & 0<\lambda \leq e_{0}, \\
\frac{\pi^{2}}{8}\left(1-\lambda^{2}\right) & \text { if } & e_{0}<\lambda \leq 1, \\
\frac{\pi^{2}\left(\lambda^{2}-1\right)}{8 \lambda^{2}} & \text { if } & 1<\lambda \leq e_{1}, \\
\max \left\{\frac{\pi^{2}\left(\lambda^{2}-1\right)}{8 \lambda^{2}}, S_{\lambda}\left(z_{0}\right)\right\} & \text { if } & \lambda>e_{1} .
\end{array}\right.
\end{aligned}
$$

Appendix B. The derivation of $\boldsymbol{d}_{\boldsymbol{\lambda}, \mathbf{3}}$ in (3.41). By (3.28), (3.32), and a direct calculation, we find

$$
W_{\lambda}(x):=\omega_{\lambda}^{-1}(x) \partial_{x}^{2} \omega_{\lambda}(x)=\frac{2 \lambda_{1}^{2}\left(3 \lambda_{1}^{2}\left(x-\lambda_{2}\right)^{2}-1\right)}{\left(1+\lambda_{1}^{2}\left(x-\lambda_{2}\right)^{2}\right)^{2}}
$$

and

$$
\partial_{x} W_{\lambda}(x)=\frac{4 \lambda_{1}^{2}\left(x-\lambda_{2}\right)\left(5-3 \lambda_{1}^{2}\left(x-\lambda_{2}\right)^{2}\right)}{\left(1+\lambda_{1}^{2}\left(x-\lambda_{2}\right)^{2}\right)^{3}} .
$$

Clearly, $W_{\lambda}\left(\lambda_{2}\right)=-2 \lambda_{1}^{2}$, and if $x$ is such that $\lambda_{1}^{2}\left(x-\lambda_{2}\right)^{2}=\frac{5}{3}$, we have $W_{\lambda}(x)=\frac{9}{8} \lambda_{1}^{2}$. Hence

$$
W_{\lambda}\left(\lambda_{2}\right) \leq W_{\lambda}( \pm 1)=\frac{2 \lambda_{1}^{2}\left(3 \lambda_{1}^{2}\left( \pm 1-\lambda_{2}\right)^{2}-1\right)}{\left(1+\lambda_{1}^{2}\left( \pm 1-\lambda_{2}\right)^{2}\right)^{2}} \leq 6 \lambda_{1}^{2}\left(\frac{\lambda_{1}\left| \pm 1-\lambda_{2}\right|}{1+\lambda_{1}^{2}\left( \pm 1-\lambda_{2}\right)^{2}}\right)^{2} \leq \frac{3}{2} \lambda_{1}^{2} .
$$

Therefore,

$$
-2 \lambda_{1}^{2} \leq W_{\lambda}(x) \leq \frac{3}{2} \lambda_{1}^{2}, \quad x \in \bar{I} .
$$

This completes the proof. $\quad \square$ 


\section{REFERENCES}

[1] M. R. Abril-Raymundo and B. Garcí-Archilla, Approximation properties of a mapped Chebyshev method, Appl. Numer. Math., 32 (2000), pp. 119-136.

[2] I. BABUŠKA AND B. GUO, Optimal estimates for lower and upper bounds of approximation errors in the p-version of the finite element method in two dimensions, Numer. Math., 85 (2000), pp. 219-255.

[3] A. Bayliss, D. Gottlieb, B. J. Matkowsky, and M. Minkoff, An adaptive pseudospectral method for reaction diffusion problems, J. Comput. Phys., 81 (1989), pp. 421-443.

[4] A. Bayliss And E. TuRkel, Mappings and accuracy for Chebyshev pseudo-spectral approximations, J. Comput. Phys., 101 (1992), pp. 349-359.

[5] C. Bernardi and Y. Maday, Spectral method, in Handbook of Numerical Analysis Vol. 5, Handb. Numer. Anal. 5, P. G. Ciarlet and L. L. Lions, eds., North-Holland, Amsterdam, 1997, pp. 209-485.

[6] J. P. BoyD, Orthogonal rational functions on a semi-infinite interval, J. Comput. Phys., 70 (1987), pp. 63-88.

[7] J. P. Boyd, Chebyshev and Fourier Spectral Methods, 2nd ed., Dover Publications, Mineola, NY, 2001

[8] C. Canuto, M. Y. Hussaini, A. Quarteroni, and T. A. Zang, Spectral Methods in Fluid Dynamics, Springer-Verlag, New York, 1987.

[9] P. G. Dinesen, J. S. Hesthaven, And J. P. Lynov, A pseudospectral collocation time-domain method for diffractive optics, in Proceedings of the Fourth International Conference on Spectral and High Order Methods (Herzliya, 1998), Appl. Numer. Math., 33 (2000), pp. 199-206.

[10] W. S. Don And D. GotTlieb, Spectral simulation of supersonic reactive flows, SIAM J. Numer. Anal., 35 (1998), pp. 2370-2384.

[11] D. FunAro, Polynomial Approximations of Differential Equations, Springer-Verlag, New York, 1992.

[12] J. E. Littlewood, G. H. Hardy, and G. Pólya, Inequalities, Cambridge University Press, Cambridge, UK, 1952.

[13] D. Gottlieb and S. A. Orszag, Numerical Analysis of Spectral Methods: Theory and Applications, CBMS-NSF Regional Conf. Ser. in Appl. Math. 26, SIAM, Philadelphia, 1977.

[14] C. E. Grosch And S. A. OrszaG, Numerical solution of problems in unbounded regions: Coordinates transforms, J. Comput. Phys., 25 (1977), pp. 273-296.

[15] B.-Y. GUO AND L. L. WANG, Jacobi interpolation approximations and their applications to singular differential equations, Adv. Comput. Math., 14 (2001), pp. 227-276.

[16] B.-Y. Guo, J. Shen, And Z.-Q. WANG, Chebyshev rational spectral and pseudospectral methods on a semi-infinite interval, Internat. J. Numer. Methods Engrg., 53 (2002), pp. 65-84.

[17] B.-Y. Guo, Jacobi approximations in certain Hilbert spaces and their applications to singular differential equations, J. Math. Anal. Appl., 243 (2000), pp. 373-408.

[18] B.-Y. GuO, J. Shen, AND Z.-Q. WANG, A rational approximation and its applications to differential equations on the half line, J. Sci. Comput., 15 (2000), pp. 117-147.

[19] B.-Y. GuO And L.-L. WANG, Jacobi approximations in certain Besov spaces, J. Approx. Theory, submitted.

[20] D. Kosloff and H. Tal-Ezer, A modified Chebyshev pseudospectral method with an o( $\left.n^{-1}\right)$ time step restriction, J. Comput. Phys., 104 (1993), pp. 457-469.

[21] W. B. Liu And J. Shen, A new efficient spectral-Galerkin method for singular perturbation problems, J. Sci. Comput., 11 (1996), pp. 411-437.

[22] W. B. LIU AND T. TANG, Error analysis for a Galerkin spectral method with coordinate transformation for solving singularly perturbed problems, Appl. Numer. Math., 38 (2001), pp. $315-345$.

[23] J. L. Mead And R. A. Renaut, Accuracy, resolution, and stability properties of a modified Chebyshev method, SIAM J. Sci. Comput., 24 (2002), pp. 143-160.

[24] J. Shen, Efficient spectral-Galerkin method I. Direct solvers of second-and fourth-order equations using Legendre polynomials, SIAM J. Sci. Comput., 15 (1994), pp. 1489-1505.

[25] J. SHEN, Efficient Chebyshev-Legendre Galerkin methods for elliptic problems, in Proceedings of ICOSAHOM'95, A. V. Ilin and R. Scott, eds., Houston J. Math., Houston, 1996, pp. $233-240$.

[26] Y. H. Song And D. Haidvogel, A semi-implicit ocean circulation model using a generalized topography-following coordinate system, J. Comput. Phys., 115 (1994), pp. 228-244.

[27] T. TAng And M. R. Trummer, Boundary layer resolving pseudospectral methods for singular pertubation problems, SIAM J. Sci. Comput., 17 (1996), pp. 430-438. 